\title{
The respiratory control mechanisms in the brainstem and spinal cord: integrative views of the neuroanatomy and neurophysiology
}

\author{
Keiko Ikeda $^{1} \cdot$ Kiyoshi Kawakami $^{2} \cdot$ Hiroshi Onimaru ${ }^{3} \cdot$ Yasumasa Okada $^{4}$. \\ Shigefumi Yokota ${ }^{5}$ Naohiro Koshiya ${ }^{6} \cdot$ Yoshitaka Oku $^{7} \cdot$ Makito Iizuka $^{3}$. \\ Hidehiko Koizumi ${ }^{6}$
}

Received: 15 March 2016/Accepted: 22 July 2016/Published online: 17 August 2016

(C) The Physiological Society of Japan and Springer Japan 2016

\begin{abstract}
Respiratory activities are produced by medullary respiratory rhythm generators and are modulated from various sites in the lower brainstem, and which are then output as motor activities through premotor efferent networks in the brainstem and spinal cord. Over the past few decades, new knowledge has been accumulated on the anatomical and physiological mechanisms underlying the generation and regulation of respiratory rhythm. In this review, we focus on the recent findings and attempt to elucidate the anatomical and functional mechanisms underlying respiratory control in the lower brainstem and spinal cord.
\end{abstract}

Keywords Respiratory rhythm $\cdot$ Pons $\cdot$ Medulla $\cdot$ Spinal cord · Parafacial respiratory group $(\mathrm{pFRG}) \cdot$ Pre-Bötzinger complex (preBötC)

Electronic supplementary material The online version of this article (doi:10.1007/s12576-016-0475-y) contains supplementary material, which is available to authorized users.

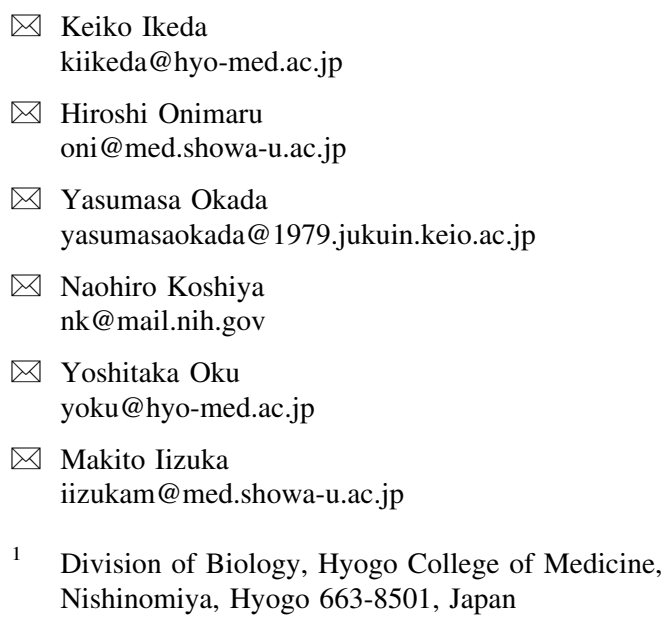

1 Division of Biology, Hyogo College of Medicine, Nishinomiya, Hyogo 663-8501, Japan

\section{Introduction}

Respiration is crucial for animal survival. In the last 10 years, the cytoarchitecture of the respiratory control center has been analyzed at the single-cell and genetic levels. The respiratory center is located in the medulla oblongata and is involved in the minute-to-minute control of breathing. Unlike the cardiac system, respiratory rhythm is not produced by a homogeneous population of pacemaker cells. Rather, it can be explained with two oscillators: the parafacial respiratory group (pFRG; Sect. 1) and the preBötzinger complex (preBötC, inspiratory pacemaker population; Sects. 2, 3). The inspiratory and expiratory activities produced in these medullary respiratory rhythm generators are modulated from various sites of the lower brainstem, including the pons (see Sect. 6) and Bötzinger complex, and are then output as motoneuron activities through the efferent networks in the brainstem and spinal cord (see Sects. 2 to 5). Different types of preparations, mainly from mice and rats, have been used to analyze

2 Division of Biology, Center for Molecular Medicine, Jichi Medical University, Shimotsuke, Tochigi 329-0498, Japan

3 Department of Physiology, Showa University School of Medicine, Shinagawa, Tokyo 142-8555, Japan

4 Clinical Research Center, Murayama Medical Center, Musashimurayama, Tokyo 208-0011, Japan

5 Department of Anatomy and Morphological Neuroscience, Shimane University School of Medicine, Izumo, Shimane 693-8501, Japan

6 Cellular and Systems Neurobiology Section, NINDS, NIH, Bethesda, MD 20892, USA

7 Department of Physiology, Hyogo College of Medicine, Nishinomiya, Hyogo 663-8501, Japan 
respiratory rhythm and pattern generation, including: medullary slice preparation in vitro (newborn or juvenile), en bloc brainstem-spinal cord preparation (newborn), decerebrated and arterially perfused preparation in situ (newborn and juvenile) and in vivo preparation (all ages). The normal respiratory motor pattern basically consists of three or four phases: pre-inspiratory, inspiratory, post-inspiratory, and late-expiratory. However, the motor output patterns in the different experimental models often display variation and the variations have caused some controversies in the field. In the last decades, new knowledge has been accumulated on the anatomical and physiological mechanisms underlying respiratory rhythm generation and regulation. In this review, we focus on these recent findings and correlate the information on the anatomical and functional mechanisms that are involved in respiratory control in the lower brainstem and spinal cord. We also introduce a novel rat line that is useful for future analyses of respiratory neural networks in vivo and in vitro. This article consists of six sections that were written by individual researchers. The focus of the sections and their respective authors are as follows: Sect. 1, the pFRG (K. Ikeda, K. Kawakami and H. Onimaru); Sect. 2, the anatomy of the preBötC (Y. Okada and S. Yokota); Sect. 3, the physiology of the preBötC (N. Koshiya); Sect. 4, the cervical circuits (Y. Oku); Sect. 5, the spinal cord (M. Iizuka); and Sect. 6, the pons ( $\mathrm{H}$. Onimaru and H. Koizumi).

\section{Section 1: Identification of the pFRG in the respiratory rhythm generator neuron complex using a novel transgenic rat line harboring Phox2b-EYFP BAC (K. Ikeda, K. Kawakami \& H. Onimaru)}

The pFRG has been named based on its position relative to the facial nucleus. It is located ventral and caudal to the facial nucleus, and predominantly consists of neurons that burst prior to inspiration [pre-inspiratory (Pre-I) neurons] [1]. The pFRG at least partially (the ventral and medial parts) overlaps the retrotrapezoid nucleus (RTN), which has been identified as an area in which neurons with projections to the ventral respiratory group (VRG) originate $[2,3]$. Thus, this region is also referred to as the pFRG/RTN. The caudal portion of the pFRG overlaps the most rostral portion of the ventral respiratory group (the Bötzinger complex), which is the ventral part of the retrofacial nucleus near the caudal end of the facial nucleus [4] and is thought to play an important role in the respiratory rhythm generation, particularly of the adult in vivo preparation $[5,6]$. This caudal portion of the pFRG corresponds to so-called rostral ventrolateral medulla (RVL) [7-9], where most Pre-I, inspiratory, and expiratory neurons have been recorded in previous electrophysiological studies.

The paired-like homeobox $2 \mathrm{~b}$ gene (Phox $2 b$ ) encodes the Phox $2 b$ transcription factor and is required for the development of a subset of cranial nerves and the lower brainstem nuclei in the central nervous system and the peripheral autonomic nervous system. The distribution of pFRG-Pre-I neurons overlaps with that of Phox $2 b-e x-$ pressing cells (Figs. 1, 2) [10-12]. It is of note that pFRGPre-I neurons in the deeper ventral medulla at the caudal area are Phox2b-negative [10].

Generally, the mouse fetal embryonic parafacial group (e-PF [13]), the rat perinatal pFRG/RTN, and the adult animal RTN $[2,14]$ are considered to be identical/corresponding populations of neurons in the different developmental stages of rodents. Indeed, the distribution and characteristics of Phox $2 b$-expressing cells in the parafacial region of the neonatal rat are basically similar to those in the adult rat $[15,16]$ and the neonatal mouse [17]. However, it is also possible that they are a discrete neuronal group that plays distinctive roles in respiratory rhythm generation during development [18-20].

In experiments using neonatal rat brainstem (medulla)spinal cord preparations, the Pre-I neurons, from which the pFRG/RTN is composed, were found to be a superior rhythm generator. It was hypothesized that they trigger inspiratory burst generation $[21,22]$ and contribute to the production of expiratory activity [23]. The pFRG/RTN functions as a chemo-sensing center that detects environmental $\mathrm{pCO}_{2}$. In a series of articles, we reported that Pre-I neurons, which are the predominant neurons in the pFRG/RTN of neonatal rats, express a paired-like homeobox $2 \mathrm{~b}$ gene (Phox $2 b)$, and that they are intrinsically $\mathrm{CO}_{2}$-sensitive [10, 24-26]. PHOX2B mutations have been described in most human cases of central congenital hypoventilation syndrome [27]. Thus, the detection of Phox $2 b$ expression in Pre-I neurons is an exciting finding because it enables us to obtain the prospect of identifying the molecules that are responsible for detecting environmental changes in $\mathrm{pCO}_{2}$. In contrast, the neurons of the pre-Bötzinger complex (preBötC) that locates just ventral to ambiguus nucleus (Amb) do not express Phox $2 b$ (Fig. 3d).

We recently reported the generation and analyses of a novel transgenic $(\mathrm{Tg})$ rat line [28]. The $\mathrm{Tg}$ rat harbors a mouse bacterial artificial chromosome (BAC) carrying a Phox $2 b$ that was modified to drive enhanced yellow 

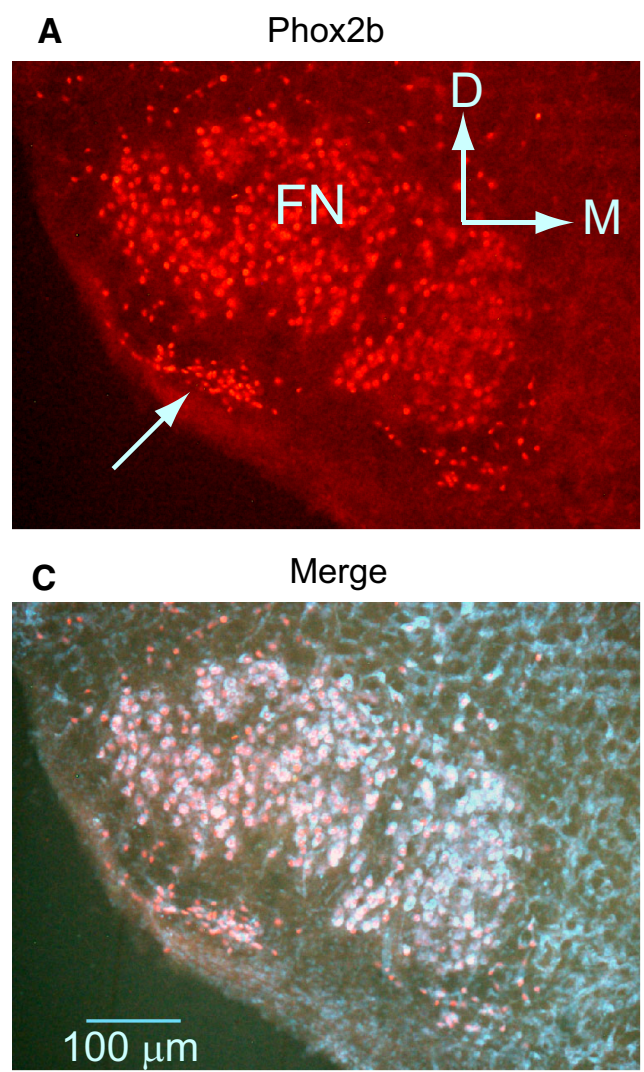

Fig. 1 Level of the transverse section used for the optical recordings (e.g., Fig. 2) and Phox $2 b$ immunoreactive cells in the most rostral medulla. a Phox2b immunoreactivity (Alexa Fluor 546). An arrow denotes a Phox $2 \mathrm{~b}$ cell cluster in the ventral parafacial region. b A NeuroTrace (435/455 blue fluorescence, Invitrogen) for Nissl staining. c A merged view of a and $\mathbf{b}$. d A ventral view of a brainstemspinal cord preparation. The preparation was cut at the level of the dotted line. AICA anterior inferior cerebellar artery, $F N$ facial

fluorescent protein (EYFP) and Cre recombinase-ER ${ }^{\mathrm{T} 2}$ (estrogen receptor T2). The Tg line shows a similar pattern of EYFP expression to that of the endogenous Phox $2 b$ in rats. Consistent with previous reports on Phox $2 b$ protein expression in rodents, the EYFP signals were mostly found in the ponto-medullary region. In the pons, the EYFPpositive neurons were found in the supratrigeminal nucleus (Su5), where they dorsally capped the motor nucleus of the trigeminal nerve (5M) (Fig. 3a, b). The $5 \mathrm{M}$ was negative for EFYP (Fig. 3b). In the medulla oblongata, EYFP-positive signals were abundant in both the spinal trigeminal nucleus (Sp5) and the pFRG/RTN (Fig. 3c). The facial motor nucleus (FN) was relatively weak and sparsely positive (Fig. 3c). The weak expression in the body of the facial nucleus has also been reported in human fetuses at 19 weeks of gestation [29]. In humans, the expression of

\section{B Nissl staining with NeuroTrace}

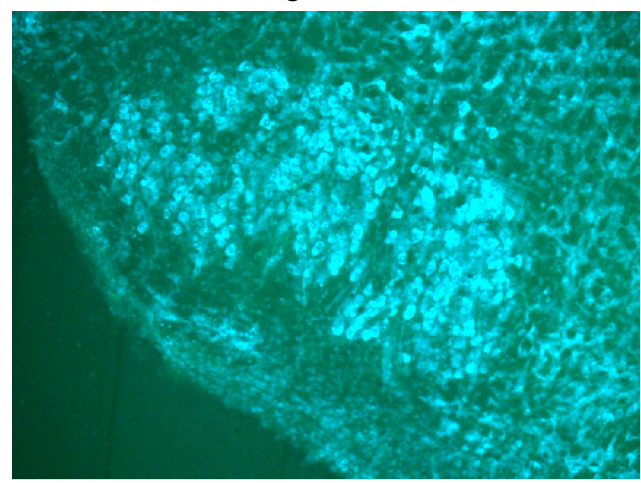

D

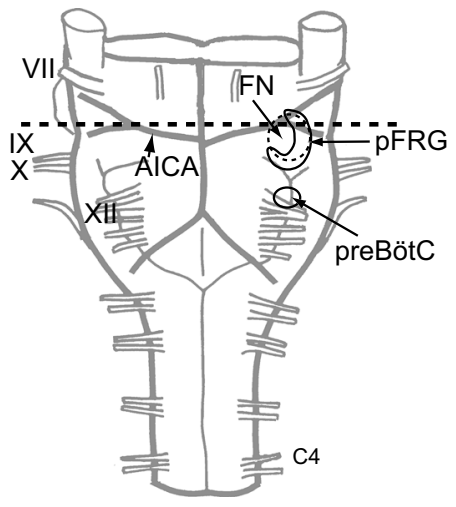

nucleus, $p F R G$ parafacial respiratory group, preBötC pre-Bötzinger complex, $V I I-X I I$ cranial nerves, $C 4$ the fourth cervical ventral root, $D$ dorsal, $M$ medial. At the level of the most rostral medulla, close to the rostral end of the facial nucleus, Phox $2 b$-ir cells formed one of the highest density clusters in the limited region ventrolateral to the facial nucleus. The cell density of the cluster was high enough to be clearly recognized, even in the Nissl-stained preparations (b)

PHOX2B in the facial nucleus disappears at later stages of gestation [15, 29]. We also observed that EYFP signals in the facial nucleus became negative over time during infancy (Ikeda and Onimaru, data not shown). In addition to the above distribution, the EYFP-positive cells are also abundant in the dorsal vagal complex, which is composed of the nucleus of the solitary tract (nTS), the dorsal motor nucleus of the vagus (dmnX), and the area postrema (AP) (Fig. 3d, e). The nucleus ambiguus (Amb) was also EYFPpositive (Fig. 3d). Interestingly, EYFP-positive cells could not be detected in the hypoglossal nucleus (Fig. 3e, 12N). The reticular nucleus (Rt) was sparsely positive for EYFP (Fig. 3c, e). We also observed EYFP-positive signals (fibers or axon terminals) through the rostro-caudal column of the ventral medulla including the preBötC and the ventral respiratory group (Fig. 1c-e). The pFRG/RTN 


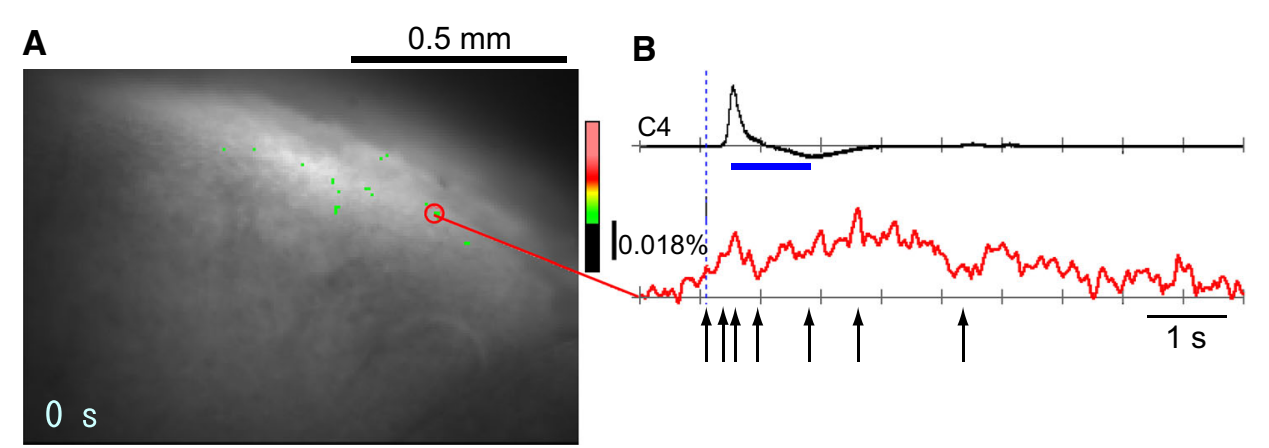

C
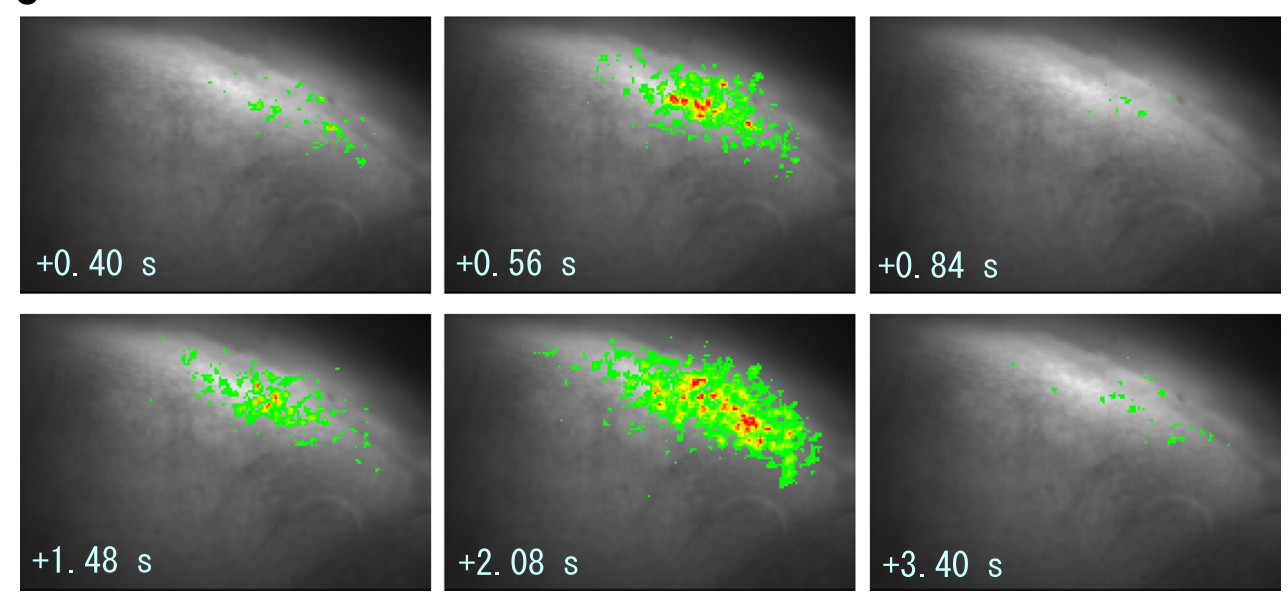

D

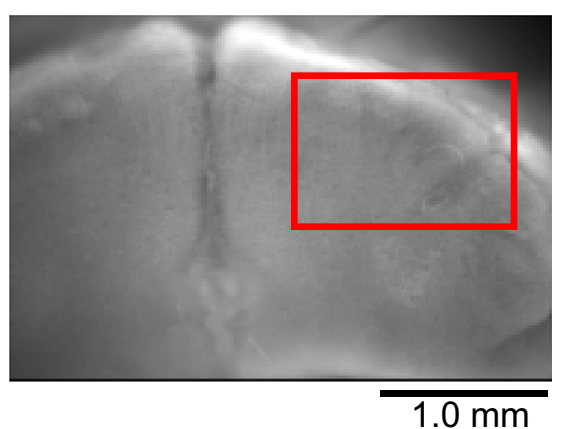

Fig. 2 Voltage imaging of the respiratory neuron activity in the ventral medulla of the rostral cut surface. The results are the averages of 40 respiratory cycles triggered by $\mathrm{C} 4$ inspiratory activity. a An optical image of the rostral cut surface; its time point is represented by the dotted vertical line on $\mathbf{b}$. b $\mathrm{C} 4$ activity and the change in fluorescence at one location (red open circle). The approximate inspiratory phase is indicated by the horizontal blue bar under the $\mathrm{C} 4$ trace. c Optical images of respiratory neuron activity. The images are arranged in a time course from left to right and top to bottom, as indicated by the numeric values, where time 0 is $\mathbf{a}$ and the subsequent images $\mathbf{c}$ correspond to time points represented by the arrows in b. d An image of the cut surface of the rostral medulla at lower
E

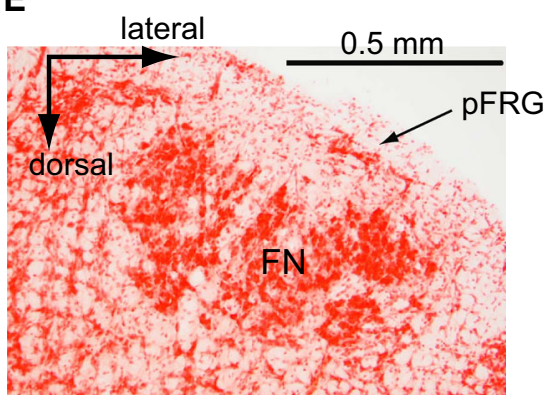

magnification. The red square denotes the area of the optical recording. e Nissl staining of the rostral cut surface after the experiment. Note that the photo clearly indicates the facial nucleus (FN) and the ventral cell cluster corresponding to the parafacial respiratory group (pFRG). Note that the optical records show the neuronal activity preceding the inspiratory activity by $500-600 \mathrm{~ms}$ in the area ventral to the facial nucleus (i.e., the rostral part of the pFRG). The activity reached its peak immediately before the peak of C4 inspiratory activity and then decreased slightly during the inspiratory phase. After the inspiratory period, the activity continued for $2-3 \mathrm{~s}$ during the post-inspiratory phase 


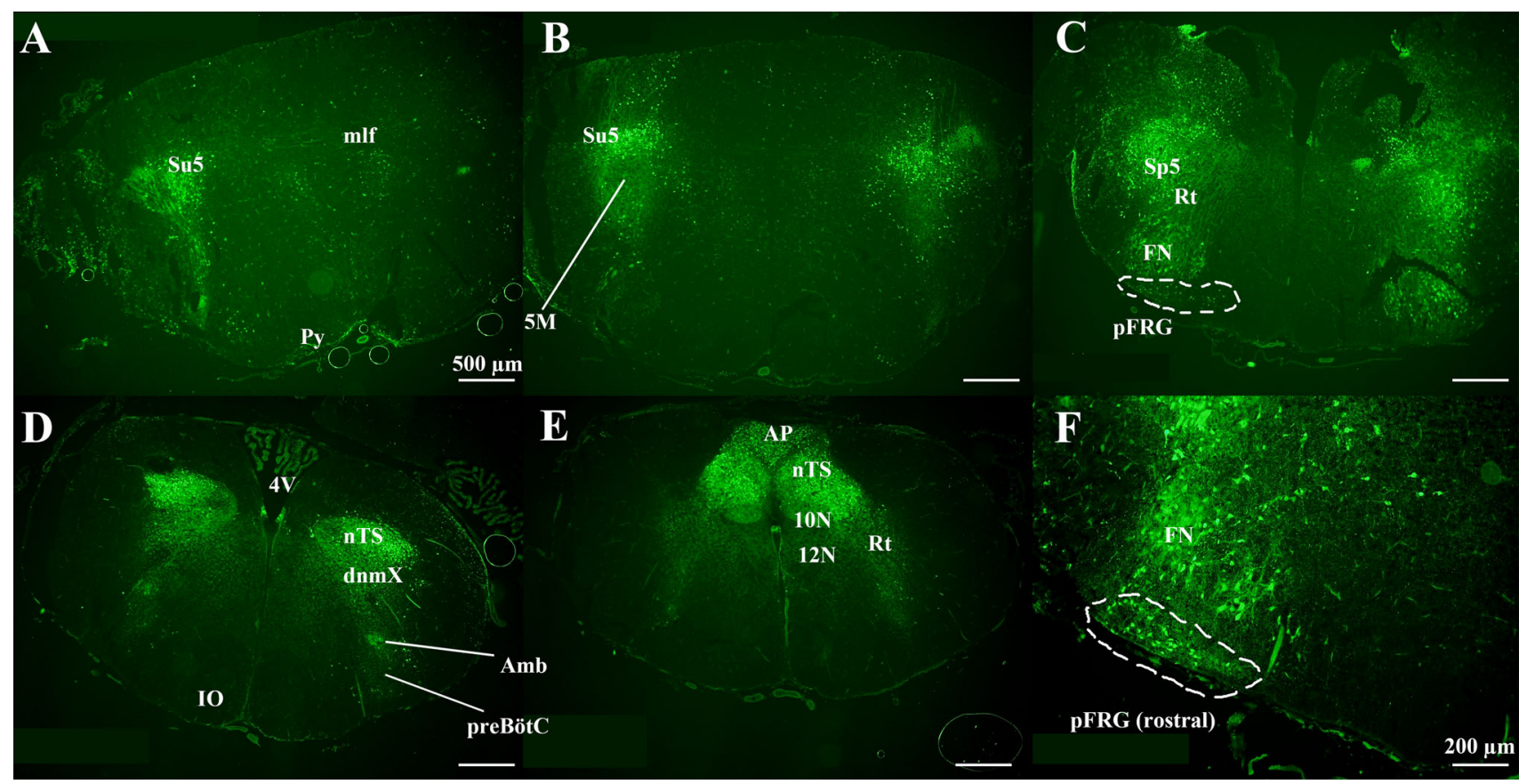

Fig. 3 Expression of enhanced-yellow-fluorescent protein (EYFP) signals driven by the mouse Phox $2 b$ enhance/promoter in the brainstem of transgenic Phox2b-EYFP/CreERT2 rats [28] from the rostral to the caudal region. In all of the micrographs, the upper side is the dorsal side. The experimental procedures are described by Ikeda et al. [28]. The experiments were performed in neonatal transgenic rats (P0-03). Su5 supratrigeminal nucleus, mlf medial longitudinal

consists of rostral (Fig. 3f) and caudal (Fig. 3c) parts [10]. The cells in the rostral pFRG/RTN contain a cluster of very superficially located neurons on the ventral side of the medulla, some of which are glutamatergic $[11,16]$. Consistent with our previous reports in which we noted that $\mathrm{CO}_{2}$-sensitive Pre-I neurons in the rostral $\mathrm{pFRG/RTN}$ are Phox2b-positive [26], all of the $\mathrm{CO}_{2}$-sensitive Pre-I neurons in the rostral pFRG/RTN that have thus far been examined in this region have been EYFP-positive [28] (data not shown). In sum, EFYP-positive signals in this rat line mirror the endogenous Phox $2 b$ expression in the neonatal stage.

Further studies to uncover the physiology of Pre-I neurons and the pFRG/RTN both in vitro and in vivo are now under investigation; e.g., for tracking the function and sole rhythmogenecity of the pFRG/RTN during the embryonic, perinatal, and adult stages of development and for the identification of the $\mathrm{CO}_{2}$ sensor molecules in the cells of pFRG/RTN through the use of fluorescence as a mark of the Pre-I neuron and of specific expression of Cre recombinase in the Pre-I neuron. fasciculus, $P y$ pyramidal tract, $5 M$ motor trigeminal nucleus, $R t$ reticular nucleus, $F N$ facial nucleus, $p F R G$ parafacial respiratory group, $4 V$ ventricle, $n T S$ nucleus of the solitary tract, $d n m X$ dorsal motor nucleus of the vagus nerve, $A m b$ ambiguus nucleus, preBöt $C$ pre-Bötzinger complex, $I O$ inferior olive, $A P$ area postrema, $10 \mathrm{~N}$ nucleus of vagus, $12 \mathrm{~N}$ hypoglossal nucleus. Scale a-e, $1.0 \mathrm{~mm}$; f $500 \mu \mathrm{m}$

\section{Section 2: The anatomy of the respiratory rhythmogenic kernel: the pre-Bötzinger complex of the medulla (Y. Okada \& S. Yokota)}

\section{The anatomical localization of the preBötC}

Basic respiratory rhythm is generated in the respiratory neuron network of the lower brainstem. In 1991, a region that is critically important for inspiratory burst generation was found within the ventral respiratory column/ventral respiratory group (VRG). This was named the pre-Bötzinger complex (preBötC) [30] (see Sect. 3). The preBötC is a small region that is bilaterally located in the reticular formation of the ventrolateral medulla. Along the rostrocaudal axis, it occupies a limited portion within the VRG (between the caudal end of the Bötzinger complex and the rostral end of the rostral VRG) [30-32]. Ventro-dorsally, it is located at a few micrometers beneath the ventral medullary surface, just ventral to the nucleus ambiguus [31, 32] (Fig. 4). Although the anatomy and function of the preBötC has mainly been studied in rodents, it has 


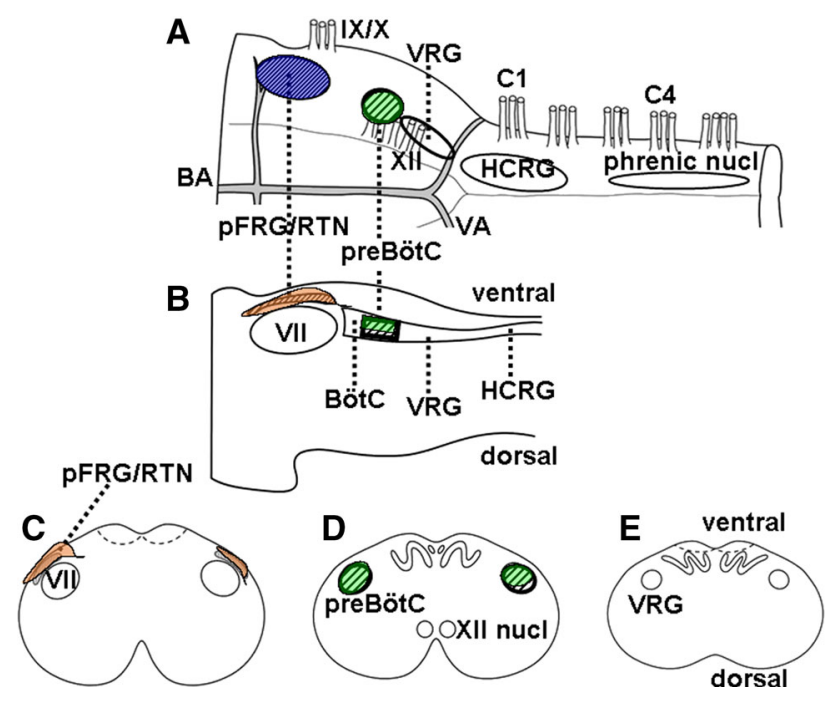

Fig. 4 Localization of the respiratory-related regions in the brainstem (the parafacial respiratory group/retrotrapezoid nucleus [pFRG/ RTN], Bötzinger complex [BötC], pre-Bötzinger complex [preBötC] and the high cervical spinal cord respiratory group [HCRG]), projected on schematic illustrations of the brainstem and spinal cord of the neonatal rat. a Ventral view. b Sagittal view. c-e Transverse view. VII facial nucleus, XII 12th cranial nerve, $C 1$ and $C 41$ st and 4th ventral roots of the cervical spinal cord, respectively, $B A$ basilar artery, $V A$ vertebral artery, $V R G$ ventral respiratory group

been identified in other animal species [33] and in humans [34].

\section{The cells composing the preBötC}

Although the preBötC is a region containing rhythmogenic cells, it does not exhibit a distinct nucleus (neuron group). The preBötC contains glutamatergic excitatory $[35,36]$ and GABAergic and glycinergic inhibitory neurons [37]. All of the neurons in the preBötC are interneurons (i.e., no motoneurons are present). The rhythmogenic neurons in the preBötC have been characterized using anatomical markers, including neurokinin-1 receptor (NK1R), somatostatin (SST), and Dbx1 [32, 36, 38-43]. Furthermore, using a combination of immunohistochemistry for the detection of NK1R and in situ hybridization for the detection of preprotachykinin A (PPTA), a precursor for substance P (SP) and a ligand for NK1R, we demonstrated the presence of PPTA mRNA-positive and NK1R-immunoreactive neurons that could play important roles in rhythm generation in the preBötC (Fig. 5a-c).

We recently reported that a subset of astrocytes in the preBötC exhibit respiratory modulating activity [44, 45]. By selectively activating astrocytes in the preBötC with an optogenetic technique, we could trigger bursting of inspiratory neurons in the preBötC, which indicates excitatory connection from astrocytes to inspiratory neurons in this rhythmogenic kernel [44]. These findings led us analyze the anatomical arrangement of neurons and astrocytes in the preBötC. We revealed that neurons and astrocytes are intimately located in the preBötC (Fig. 6) and that the astrocytic processes exhibit contact with SSTergic neurons (data not shown), which is also in agreement with our physiological report that astrocytes and rhythmogenic neurons are functionally coupled in the preBötC [44]. The role of preBötC astrocytes in respiratory rhythmogenesis could be active and should be further investigated in the future (also see Sect. 3).

\section{Neuronal projection to the preBötC neurons}

Neurons in the preBötC are functionally coupled with other respiratory-related regions in the brainstem. To investigate the anatomical connections, we conducted retrograde and anterograde tract tracing, and revealed that the NK1R- and SST-immunoreactive neurons in the preBötC regions receive axon terminals from the contralateral preBötC. The axon terminals from the preBötC make asymmetrical, putative excitatory, synaptic contact with the NK1R-immunoreactive neurons in the contralateral preBötC [36]. We also combined retrograde tracing by injecting fluorogold into the unilateral preBötC with in situ hybridization to detect PPTA, and demonstrated that the putative rhythmogenic PPTA mRNA-positive neurons in the preBötC project to the contralateral preBötC (Fig. 5d-h). It has also been reported that SP and enkephalinergic axon terminals form synapses on NK1Rimmunoreactive neurons in the preBötC [46]. These findings suggest that the connection between bilateral preBötC neurons, especially SPergic commissural neurons, serves to synchronize the timing of the oscillatory activities in the bilateral preBötC.

\section{Neuronal projection from the preBötC neurons}

With respect to projection to other brain stem regions, neurons in the preBötC send axonal fibers to various respiratory-related regions. Tan et al. [47] demonstrated the axonal projection of SSTergic neurons in one side of the preBötC to the bilateral Bötzinger complex, VRG regions caudal to the preBötC, parafacial respiratory group/retrotrapezoid nuclei, parabrachial/Kölliker-Fuse nuclei and periaqueductal gray region. Furthermore, we showed that the neurons in one side of the preBötC region send axonal projections to the bilateral hypoglossal premotor areas, the 

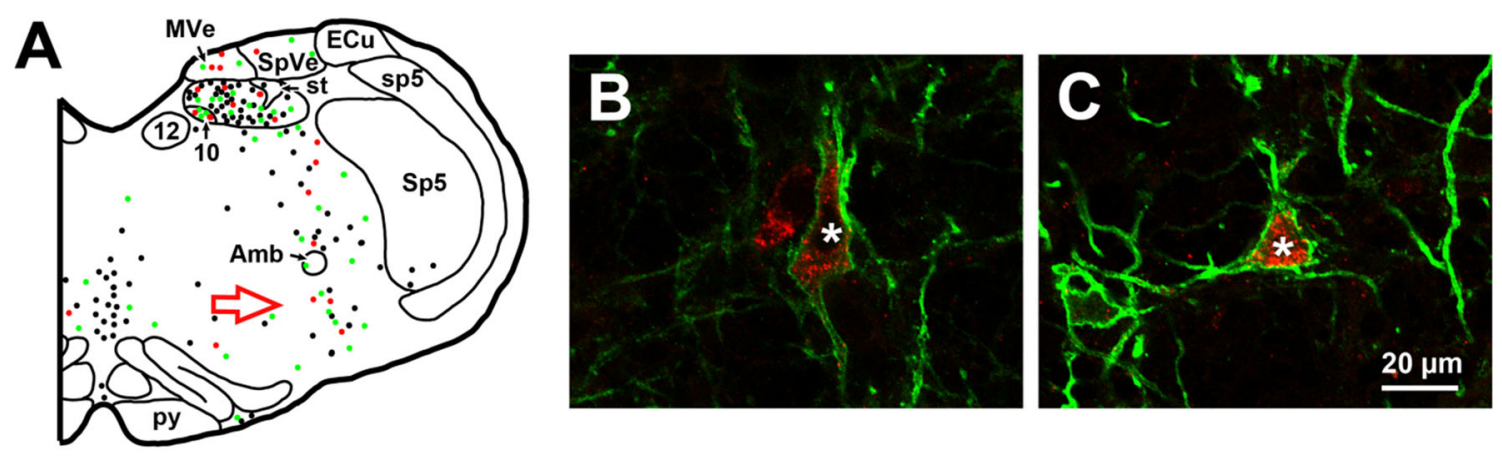

D
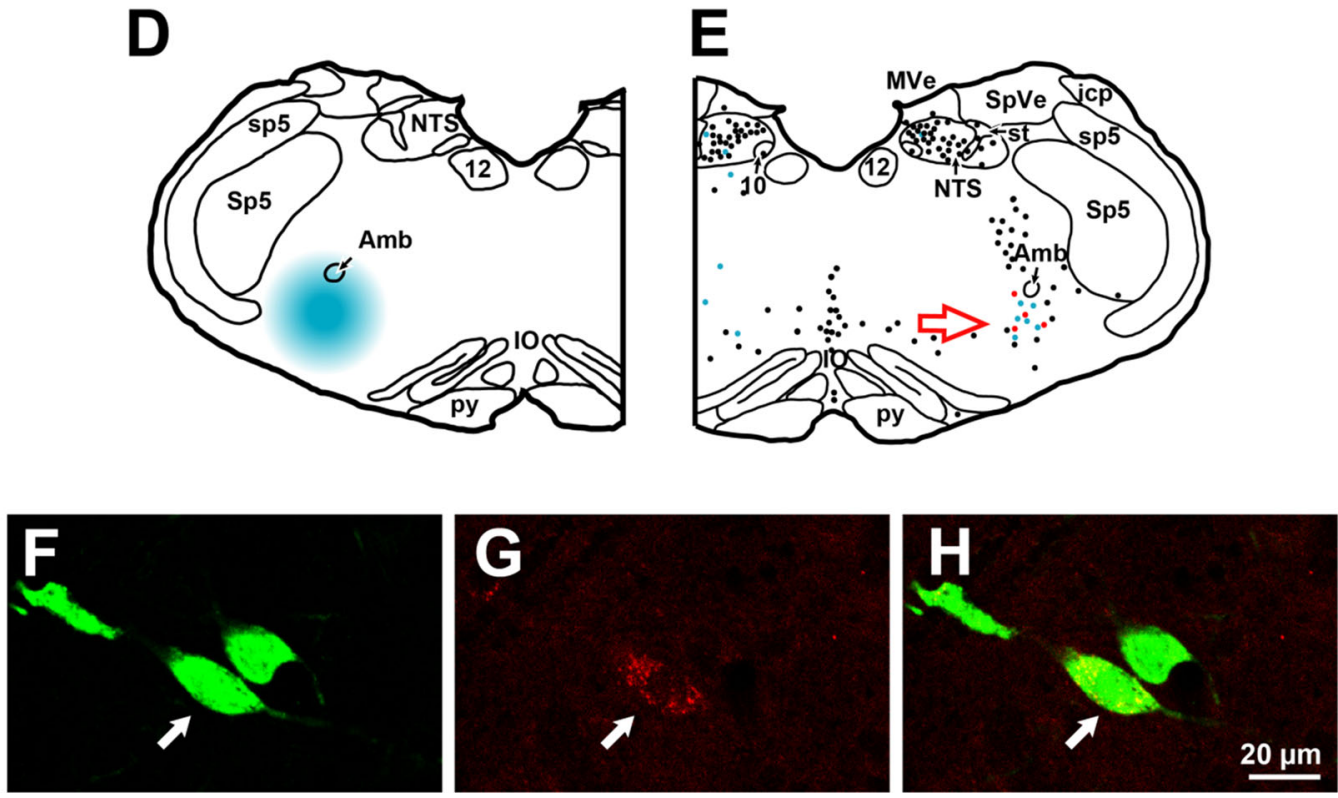

Fig. 5 Localization of anatomically identified putative rhythmogenic neurons in the pre-Bötzinger complex (preBötC). a Line drawings showing the distribution of neurokinin 1 receptor (NK1R)-immunoreactive neurons (green dots), preprotachykinin A (PPTA) mRNApositive neurons (black dots), and double-labeled neurons (red dots) in the medulla. b, c Confocal images showing the appearance of PPTA mRNA-positive neurons (red) and NK1R-immunoreactive neurons (green) in the preBötC. The asterisks indicate double-labeled neurons. d The preBötC region where retrograde tracer fluorogold (FG) was injected (shaded area). e The distribution of FG-labeled neurons (blue dots), PPTA mRNA-positive neurons (black dots), and PPTA mRNA-positive neurons simultaneously labeled with FG (red

bilateral hypoglossal motor nuclei and the bilateral nuclei tractus solitarius [36]. However, there are no reports indicating the direct projection from preBötC neurons to either the cerebellum or the spinal cord [48]. In contrast, the putative rhythmogenic neurons in the preBötC receive glutamatergic projections, e.g., from the lateral periaqueductal gray region [49] and the parabrachial nucleus (unpublished observation).

In conclusion, so far, elucidated anatomy of the preBötC suggests that it plays a critically important role in dots) in the contralateral medulla. The open arrows in (a) and (e) indicate the preBötC region. $\mathbf{f}-\mathbf{h}$ PPTA mRNA-positive preBötC neurons with projection to the contralateral preBötC. Confocal images of FG-labeled (f) and PPTA mRNA-positive (g) neurons. The merged image is shown in $\mathbf{h}$. The arrow indicates a double-labeled neuron. 10 dorsal motor nucleus of vagus, 12 hypoglossal nucleus, $A m b$ nucleus ambiguus, $E C u$ external cuneate nucleus, icp inferior cerebellar peduncle, $I O$ inferior olive, $M V e$ medial vestibular nucleus, NTS nucleus of the solitary tract, $p y$ pyramidal tract, $S p 5$ spinal trigeminal nucleus, $s p 5$ spinal trigeminal tract, $S p V e$ spinal vestibular nucleus, $s t$ solitary tract

rhythmogenesis among the various respiratory networks, although it is quite probable that multiple respiratory networks distributed throughout the higher and lower brainstem and the high segments $(\mathrm{C} 1-\mathrm{C} 2)$ of the spinal cord are interconnected and are together involved in respiratory rhythm generation in the in vivo condition [33]. It is necessary to further investigate the anatomy of the preBötC, including the local connection between astrocytes and neurons, to better understand the physiological and pathophysiological mechanisms of respiratory rhythm generation. 

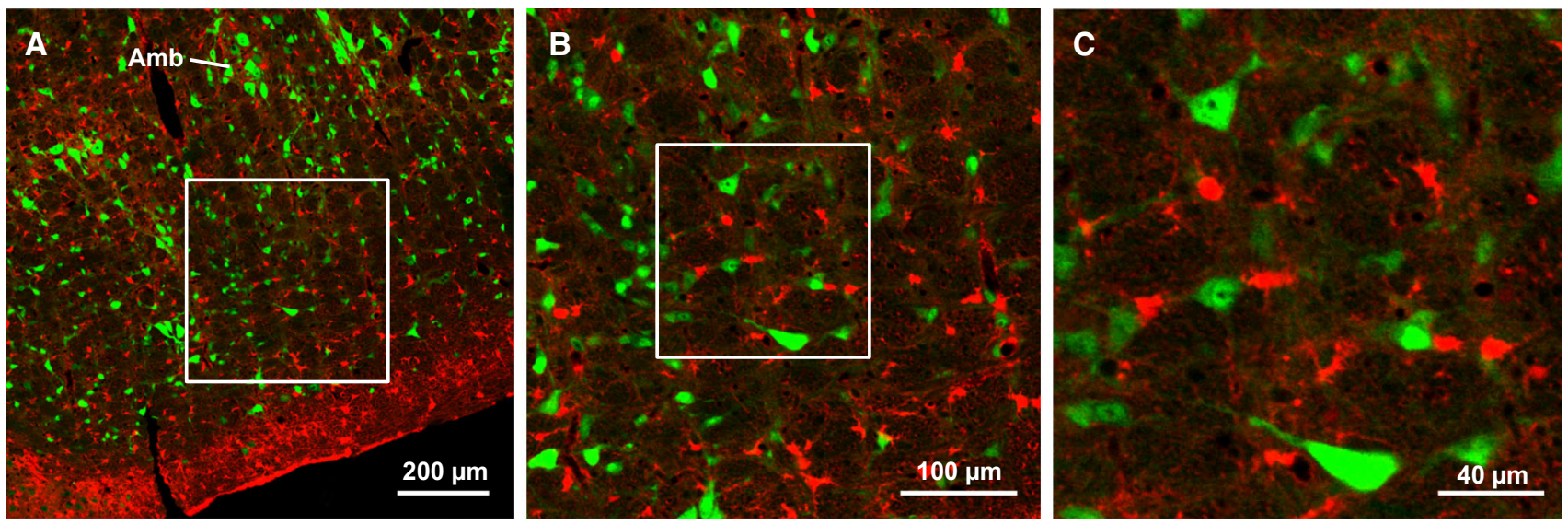

Fig. 6 Colocalization of neurons and astrocytes in the ventrolateral medulla. Neurons and astrocytes are identified as neuron-specific marker NeuN-positive cells (green) and astrocyte-specific marker S100b-positive cells (red), respectively. a Ventrolateral medullary region. The square indicates the preBötC region, and corresponds to

\section{Section 3: The physiology of the pre-Bötzinger complex from a rhythmogenic perspective $(\mathbf{N}$. Koshiya)}

\section{Functional localization}

The pre-Bötzinger complex (preBötC) was discovered as the region that generates respiratory rhythm in neonatal rodent brainstem-spinal cord preparation in vitro. Smith et al. [30] demonstrated that efferent rhythmic respiratory motor activities persist, essentially unchanged, in the spinal ventral roots that serve the phrenic $(\sim \mathrm{C} 4)$ and thoracic intercostal nerves, until live coronal sectioning by a vibratome from the rostral end of the brainstem encroached on the rostrocaudal level near the rostral-most hypoglossal (XII) nerve rootlet exit (see Sect. 2 for more detailed anatomy). This level was also crucial when vibratome sectioning was performed from the caudal end of the brainstem (while recording inspiratory motor nerve activities in cranial nerves such as the XII and/or glossopharyngeal nerves). These two-way deletion experiments, combined with electrophysiological recordings [30], allowed for the respiratory rhythmogenic kernel of the preBötC to be functionally located. Transverse brainstem slice preparations cut at this level maintained a vital rhythm in the XII activities; i.e., the preBötC, XII premotor microcircuits, and its motoneurons, motor axons, and nerve rootlets were miraculously located within the same coronal plane and were captured in a single thin slice. This allowed for the creation of highly reduced, yet functionally complete, breathing slice preparations. In 2014, the first whole slice activity imaging confirmed its localization and the associated regions (Fig. 7; Movie 1) [36]. the area in b. b An enlarged image of the preBötC. The square indicates the area in c. c A high-magnification picture showing colocalized cell bodies of neurons and astrocytes. Amb nucleus ambiguus

\section{The pacemaker neurons and their significance}

Extracellular recording has shown that the rhythmic bursting of some preBötC inspiratory neurons can continue after the attenuation of synaptic transmissions with low calcium medium in slices in vitro [50]; however, neither cellular biophysics nor synchronization mechanisms could have been studied before functional imaging, optical preidentification, and visualized patch-clamp recording were employed [35]. Since the breathing rhythm is bilaterally synchronous, the existence of direct reciprocal connections was hypothesized. This was successfully demonstrated by labeling the preBötC rhythmogenic neurons via their contralaterally projecting axons. Calcium-sensitive dyes injected directly into one side of the preBötC had labeled some contralateral preBötC neurons; however, the signal was mostly static, probably because the vascular uptake and transport had kept the dye isolated from the calciumdynamic cytosolic domains. The microinjection of membrane semipermeable acetoxymethyl ester (AM) dyes around the (hypothetical) axons en passant worked. Within the heterogeneous reticular formation, where no circumscribed nucleus is visible, some of the labeled neurons (flashers) that were restricted to a region that corresponded to the preBötC showed transient fluorescence that was synchronous to XII inspiration. Half of the inspiratory flashers had intrinsic rhythmogenicity (pacemakers) when they were functionally isolated from the rest of the population with an AMPA/KA glutamatergic transmission blocker (CNQX). The yield of pacemakers in other exogenous preidentification experiments that were subsequently performed using NK1R-mediated live fluorescence labeling was $25 \%$ [51]. The pacamakers' burst rate was a 
Pan-slice activity mapping

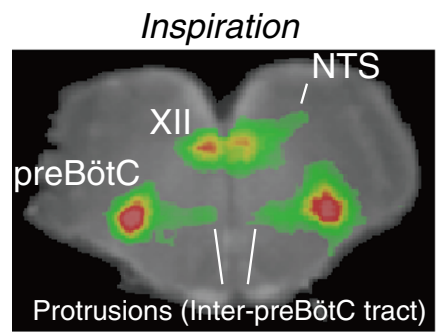

Instantaneous activity within a preBötC and its $C O A$
Functional connectomics by membrane potential movies
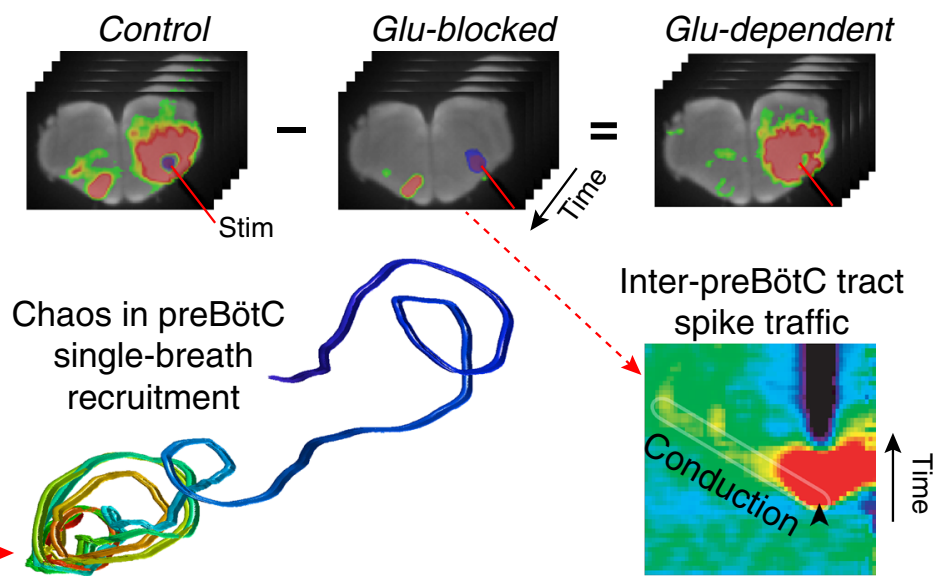

Fig. 7 Functional connectomics of the preBötC [36]. Panels corresponding to the present paragraphs: functional localization (pan-slice activity mapping); instantaneous activity $(d t=500 \mu \mathrm{s})$ and COA (black ball); Single-breath recruitment chaos of the preBötC population; Inter-preBötC tract's action potential conduction under CNQX

monotonic function of their membrane potential [35] until the membrane potential exceeded the oscillatory regime, at which time they became tonically active; the system would then shift into an inspiratory apnea. This was the first cellular mechanism identified that may underlie the system's respiratory rhythm. With tonic (DC) afferent information such as chemoreceptor feedback, it modulates the frequency (FM) of the rhythmic motor drive and thus ventilation. The central homeostatic control was dissected down to the cellular biophysical level.

\section{Persistent sodium conductance (gNaP)}

$g N a P$, in combination with leak conductance, is found to be crucial to the pacemaker inspiratory neuron functions. There are three distinct functions in its rhythmic burst generation. The first is burst formation. Its "persistence" is essential to burst generation. The neurons use their $\mathrm{N}$-shaped $I-V$ relationship [52,53] where, within the negative slope domain, the cell would recursively auto-depolarize into a burst (i.e., a train of action potentials during a depolarization in a $10^{-1}-10^{0}$-s time scale. The burst termination is the second function that is achieved by cumulative high-voltage-sensitive inactivation of $\mathrm{gNaP}$ at every action potential through the burst until spike generation is no longer possible. The third is its rhythm determination. It is not their persistency (a hard-to-inactivate nature); it is rather their characteristically slow disinactivation $\left(10^{0}-10^{1}\right.$-s scale recovery from burstinduced inactivation and silence), which is likely to be a determinant of the interburst interval of the pacemaker neurons [54]. This $g N a P$ could be blocked with low concentrations of its blockers [riluzole $(5 \mu \mathrm{M})$ or tetrodotoxin
(Glu-blocked); and Glutamatergic premotor relays (Glu-dependent). See the open-access online graphic abstract of the paper for further details: http://www.sciencedirect.com/science/article/pii/S030645221 4002085

$(5 \mathrm{nM})]$ when they were precisely microinfused into preBötC in slice preparations, superfused over slice preparations with the caudal cut surface of the preBötC exposed [53] (the rostral side contains bilaterally synchronizing axons [55]), or transarterially perfused into brainstem spinal cord in situ preparations without rostral structures beyond the preBötC [56], which would cause the network to become incapable of generating rhythmic inspiration (as well as gasping [57]).

\section{Leak conductance (gLeak)}

While it is referred to as "leak," ligand-gated channel conductances are often ohmic and not voltage gated, and can thus function as a linear gLeak. This includes protonsensitive channels, which may be responsible for the chemosensitivity of the preBötC cells (e.g., TASK channel [58]). As was predicted theoretically [54], a quantitative balance of $g N a P$ and gLeak was found to distinguish pacemakers from non-pacemakers (see Fig. 5 of Del Negro et al. [52]).

\section{Calcium pacemaking}

Pacemaking primarily on calcium-gated non-specific cationic conductance $(g C A N)$ has also been suggested $[59,60]$. While calcium influxes are not required for $\mathrm{gNaP}$ based pacemaking [61], gNaP blockers can reproducibly block slice pacemaking, (see $g N a P$ paragraph above), and the termination mechanism for $g C A N$-based burst is unknown, the contribution of calcium to other types of cell bursting remains possible. 


\section{Synaptic synchronization}

The glutamatergic synchronization of the pacemaker and other inspiratory neurons is crucial for the inspiratory activity formation at neural population levels. This was demonstrated by an optical simultaneous multi-neuron activity recording using retro-axonal calcium dye labeling [35]. Its dependency on glutamatergic transmission was proven by the desynchronization of the activity of synchronously active inspiratory neurons after the administration of a glutamatergic antagonist (CNQX).

\section{Population recruitment chaos}

The single-breath recruitment of the preBötC population dynamics was discovered using high-speed $(2 \mathrm{k} \mathrm{fps})$, voltage-sensitive dye imaging (VSDI; see Fig. 8 of Koshiya et al. [36]). The spatiotemporal location of the instantaneous center of activity "mass" (COA) was tracked and reduced to its velocity sequence, which was discovered to be chaotic (i.e., not fully periodic yet deterministic). This chaos at the origin of activity may underlie the respiratory chaos in systems in which the activity is not identically recursive yet deterministic [52, 62].

\section{The bilaterally synchronizing tract}

The inter-preBötC tract was not anatomically distinct but was found to be a functional structure by a pan-slice, highspeed imaging procedure [36], in which compound action potentials were spatiotemporally tracked from one side of the preBötC during the microstimulation of the other side. The suppression of the recursive autoactivation of the bilateral CNQX population was used to unveil the one-shot traffic (Fig. 7 Glu-blocked and Movie 2b; also see Fig. 7 of Koshiya et al. [36]). To the best of our knowledge, this was the first and remains the only visualization of compound action potential conduction through the brain tract of vertebrates, which is different from general activity propagation where rapid dynamics such as return of activity to the baseline are not observed (Movie 2).

\section{Premotor synaptic cascades}

Glutamatergic premotor relays were demonstrated in the full network configurations from one side of the preBötC to the bilateral XII motor nuclei using movie subtraction: [control movie] - [CNQX movie] (Fig. 7 Control and Glu-blocked; Movie 2ab) where CNQX-insensitive (thus glutamatergic) depolarization was extracted through premotor areas (Fig. 7 Glu-dependent; Movie 2c). Premotor neurons have been found between the preBötC and XII areas [63]. The study located the areas that were wholly synaptically involved, including the contralateral premotor areas [36].

\section{Non-glutamatergic populations}

Non-glutamatergic cells in the preBötC include inhibitory neurons (GABAergic and glycinergic, ([37], [55]; see Sect. 2 for other phenotypes). There were no inhibitory bursters [55] (the contradictory study [64] could have shown loss of either periodic synaptic drives [32, 52] or multi-cell synchrony [32]) and the kernel rhythm persisted after the inhibitory transmission blockade [65]. They may shape and/or tune the inspiratory activities in vitro.

Some of the astrocytes in the preBötC also showed calcium transients during inspiration [44]; occasionally with a pre-inspiratory rise. While some remained oscillatory after TTX, they lost synchrony and their individual rhythms slowed by an order of magnitude. Their burst timings had therefore been set by the neurons. The biophysical interactions between the inspiratory neurons and the inspiratory astrocytes are yet to be determined.

\section{The relevance to the field of physiology}

The preBötC is the only population oscillator in the mammalian motor center, where the rhythmogenic mechanisms have been demonstrated at the cellular biophysics and neural population levels if not yet fully. These wideranging and convergent investigational approaches may inspire other motor center studies.

\section{Section 4: The structure and function of the respiratory neuronal circuits of the high cervical spinal cord (Y. Oku)}

\section{The localization and connectivity of the cervical respiratory neurons}

The localization and the synaptic connections of the spinal respiratory neurons were extensively investigated in the late 1980s and early 1990s. It was motivated by the hypothesis that the net depolarization provided by the monosynaptic bulbospinal projections from medullary inspiratory neurons only accounted for a small fraction of the total amount that would be necessary for phrenic or intercostal motoneuronal discharge [66], which implies the existence of interneurons that relay the medullary respiratory drive to spinal motoneurons. Lipski and Duffin [67] demonstrated the presence of a longitudinal column of inspiratory neurons extending from the caudal nucleus retroambigualis to the rostral segment of the $\mathrm{C} 3$ in cats. These upper cervical inspiratory neurons (UCINs) were 
located around the intermediate zone (lamina VII) of the gray matter, and projected into the vicinity of the phrenic and intercostal motoneurons [67, 68]. Nakazono and Aoki [69] demonstrated that UCINs have excitatory mono- or paucisynaptic connections with the ipsilateral phrenic motoneurons, supporting the hypothesis that UCINs function as a propriospinal respiratory system, similarly to a propriospinal locomotor system [70]. Palisses et al. [71] reported a different inspiratory interneuron pool at the $\mathrm{C} 3$ C6 spinal cord levels, approximately $500 \mu \mathrm{m}$ dorsal to the phrenic motor nucleus.

\section{The rhythmogenesis of the cervical respiratory neuronal circuits}

Animals spinalized at the $\mathrm{C} 1-\mathrm{C} 2$ levels generate spontaneous rhythmic phrenic activity [72-74]. In 1977, Coglianese et al. [73] observed spontaneous rhythmic phrenic nerve activity in $\mathrm{C} 1-\mathrm{C} 2$ spinalized non-paralyzed dogs following the administration of a respiratory stimulant, doxapram hydrochloride. A few years later, Aoki et al. [72] observed the temporary recovery of periodic phrenic motoneuron activity, approximately $1 \mathrm{~h}$ after spinomedullary transection in cats. However, since the administration of a neuromuscular transmission blocking agent, curare abolishes phrenic activity, respiratory rhythmicity is thought to be supported by feedback inputs from cutaneous and chest wall proprioceptors. Viala et al. [74] observed synchronous short-lasting and long-lasting rhythmic bursts on the phrenic nerves after $\mathrm{C} 2$ level spinal transection in curarized and vagotomized rabbits after the administration of DOPA in combination with a monoamine oxidase inhibitor, nialamide. They suggested that short-lasting bursts are driven by the forelimb and hindlimb locomotion generator, whereas long-lasting bursts are driven by the spinal respiratory rhythm generator because they are enhanced by hypercapnia independently from the locomotor bursts. Similar long-lasting synchronous respiratory activity of a spinal origin can be recorded in the in vitro brainstem spinal cord preparation from rats by the pharmacological activation of deep diethyl ether anesthesia [75]. This long-lasting spinal respiratory activity was found to coexist with the medullary respiratory activity, and persist after $\mathrm{C} 1$ spinal transection. Based on the transection experiments, the authors concluded that the spinal respiratory rhythm generator is located in the $\mathrm{C} 4$ and $\mathrm{C} 6$ spinal segments. However, in a later analysis [76], it was revealed that the long-lasting activity was exclusively recorded from cervical ventral roots and never observed in the cranial or phrenic nerves. Moreover, only non-respiratory motoneurons exhibited rhythmic depolarization in phase with the long-lasting bursts. Thus, it is unlikely that the long-lasting activity is involved in respiratory function. Recently,
Kobayashi et al. [77] demonstrated that $\mathrm{C} 1 / \mathrm{C} 2$ spinal slices from neonatal mice are capable of generating rhythmic bursts. Spontaneous burst activity in the $\mathrm{C} 1 / \mathrm{C} 2$ ventral roots occurred shortly (approximately $30 \mathrm{~min}$ ) after transection and remained stable in the cervical slice for approximately $30 \mathrm{~min}$ and gradually deteriorated to cessation in $1-2 \mathrm{~h}$.

\section{Revisiting the cervical respiratory neurons}

Optical imaging using voltage-sensitive dyes led to the discoveries of novel respiratory regions: the parafacial respiratory group [78] and the high cervical respiratory group (HCRG) in the spinal cord [79]. The HCRG is distinct from the UCIN because it is located in the ventral portion of the ventral horn at the level of the spinomedullary junction with the $\mathrm{C} 2$ segment. The HCRG consists of interneurons and motoneurons, which are responsive to both respiratory and metabolic acidosis, suggesting that it forms a propriospinal respiratory neuronal network [80]. The discovery of the HCRG motivated the re-examination of the role of the spinal cord in respiratory rhythmogenesis. Jones et al. [81] recorded phrenic (PNA) and hypoglossal (HNA) nerve activity in perfused brainstem preparation from rats to examine the effects of the transverse sectioning of the high spinal cord. Transverse transections at the pyramidal decussation not only immediately abolished PNA but also resulted in a progressive decline in the HNA amplitude and rhythm. Transverse transections at the first cervical spinal segment level did not abolish HNA rhythmicity. These results indicate the importance of the structures at the spinomedullary junction in eupneic respiration. In the current concept, eupnea, which is defined by a breathing pattern of inspiration, post-inspiration, and expiration, requires the integrity of the pontine-medullary respiratory network [56]. The essential structures for eupneic breathing have been hypothesized to extend from the pons to the pre-Bötzinger complex; structures caudal to the obex are thought to be unnecessary for eupneic breathing. However, the observation of Jones et al. [81] contradicts the current concept of the genesis of eupnea.

\section{Respiratory regeneration after spinal cord injury}

In vivo and in vitro observations suggest that the spinal center of respiratory rhythm generation takes $1-12 \mathrm{~h}$ to activate after intervention [72, 73, 77]. This may suggest that a certain recovery time is necessary for the network plasticity to reorganize the spinal respiratory neuronal circuits after injury. The propriospinal respiratory network may compensate for functional deficits by either activating auxiliary pathways or by serving as a backup respiratory rhythm generator. With the help of these backup 
mechanisms, endogenous sprouting or synaptogenesis would be able to repair the injured pathway to restore function. Indeed, the photoactivation of channelrhodopsin in the motoneurons, glia, and spinal interneurons at the level of the phrenic motor nucleus was able to functionally restore hemi-diaphragm activity 4 days after $\mathrm{C} 2$ hemisection [82]. After cervical spinal cord injury, chondroitin sulfate proteoglycans around the phrenic motor neurons are involved in the upregulation of the perineuronal net. The digestion of these potently inhibitory extracellular matrix molecules with Chondroitinase $\mathrm{ABC}$ (ChABC) promotes the plasticity of the spared tracts and restores activity to the paralyzed diaphragm [83]. The implantation of an autologous peripheral nerve graft (PNG), in combination with the addition of $\mathrm{ChABC}$, allows for additional axonal regeneration through the PNG, leading to recovery from impaired function [83]. The transection of the PNG after recovery caused an unusual increase in tonic EMG activity, which would have originated from the activity of propriospinal neurons that innervate the motor neurons. These results suggest that propriospinal neurons, which are recruited by regenerating axons, play an important role in circuit reorganization.

In conclusion, although the high spinal cord is not the site of the primary respiratory rhythm generator, it is involved in shaping eupneic breathing patterns, and in the event of injury, it recruits auxiliary pathways for circuit repair and reorganization, and may even exert as a backup respiratory rhythm generator. Thus, the respiratory neuronal circuits of the high cervical spinal cord are a vital constituent of the respiratory network.

\section{Section 5: The involvement of spinal interneurons in the generation of the rostrocaudal gradient of intercostal inspiratory motor activity (M. Iizuka)}

Respiration involves a complex pattern of movements for which numerous motoneurons distributed along the spinal cord need to fire in the proper spatial and temporal sequences. A number of studies involving electrical recordings from intercostal muscles or nerves in anesthetized cats $[84,85]$, decerebrated cats [86], anesthetized dogs [87, 88], and humans [89], have shown that the external intercostal muscles, or their nerve filaments, are active during inspiration, and that the inspiratory activities in the rostral interspaces are stronger than those in the caudal interspaces (see De Troyer et al. [90] for review). Similarly, the parasternal region of each of the interchondral internal intercostal muscles (the so-called parasternal intercostals) is active during the inspiratory phase, and muscles in the rostral interspaces show stronger activities than muscles in the caudal interspaces in anesthetized dogs and awake humans [91, 92]. Deafferentation of the rib-cage does not affect the rostrocaudal gradient of the inspiratory motor activity in the parasternal intercostals [91]. Similarly, in isolated brainstem-spinal cord preparations from the neonatal rat, which has no afferent feedback, the inspiratory activities in the more rostral thoracic ventral root were found to be larger than those in the caudal thoracic ventral root [93]. These studies suggest that the central respiratory networks organize this basic spatial and temporal pattern for respiration.

A histochemical study in the cat demonstrated that the composition of fiber types in the parasternal intercostals is similar between the thoracic spinal segments, suggesting the mean and dispersion of the size of the parasternal intercostal motoneuron are similar between the segments [80]. The external intercostals in the rostral thoracic segments have a larger ratio of the slow-twitch oxidative type to the fast-twitch types [80], suggesting that the mean size of the external intercostal motoneurons shifts to smaller in the more rostral segments. In the hindlimb muscle, although the soma size of the slow-twitch oxidative motor unit tended to be smaller than that of the fast-twitch motor unit, the sizes were largely overlapped [94]. At present, the size and number of inspiratory motoneurons in each segment of the thoracic spinal cord have not been studied in detail. In the following, we assumed that the inspiratory motoneurons are uniformly distributed along the thoracic spinal cord, and discussed how the central respiratory networks organize the rostrocaudal gradient of intercostal inspiratory motor activity.

The possibilities were roughly divided into two: the spinal interneurons are involved or they are not. In cases where the spinal interneurons are not involved, the bulbospinal inspiratory neurons and motoneurons organize the rostrocaudal gradient. Three ways would be possible. The first is the excitatory bulbospinal neurons project richly to the inspiratory motoneurons in the rostral segments of the thoracic spinal cord (Fig. 8a). The second is the synapses from the excitatory bulbospinal neurons to the more rostral inspiratory motoneurons terminate on the more soma side, and evoke a larger excitatory postsynaptic potential. The third is that the motoneurons in the rostral thoracic cord have a lower threshold than the motoneurons in the caudal thoracic cord. These three ways as described above are not exclusive to each other.

It is well documented that the bulbospinal neurons provide monosynaptic inputs to the intercostal motoneurons $[66,95,96]$. Thus far, all of the detected monosynaptic connections have been excitatory. Although Davies et al. [66] showed that external intercostal inspiratory activity was directly relevant to the rostrocaudal gradient, they provided no evidence to suggest that the inspiratory 


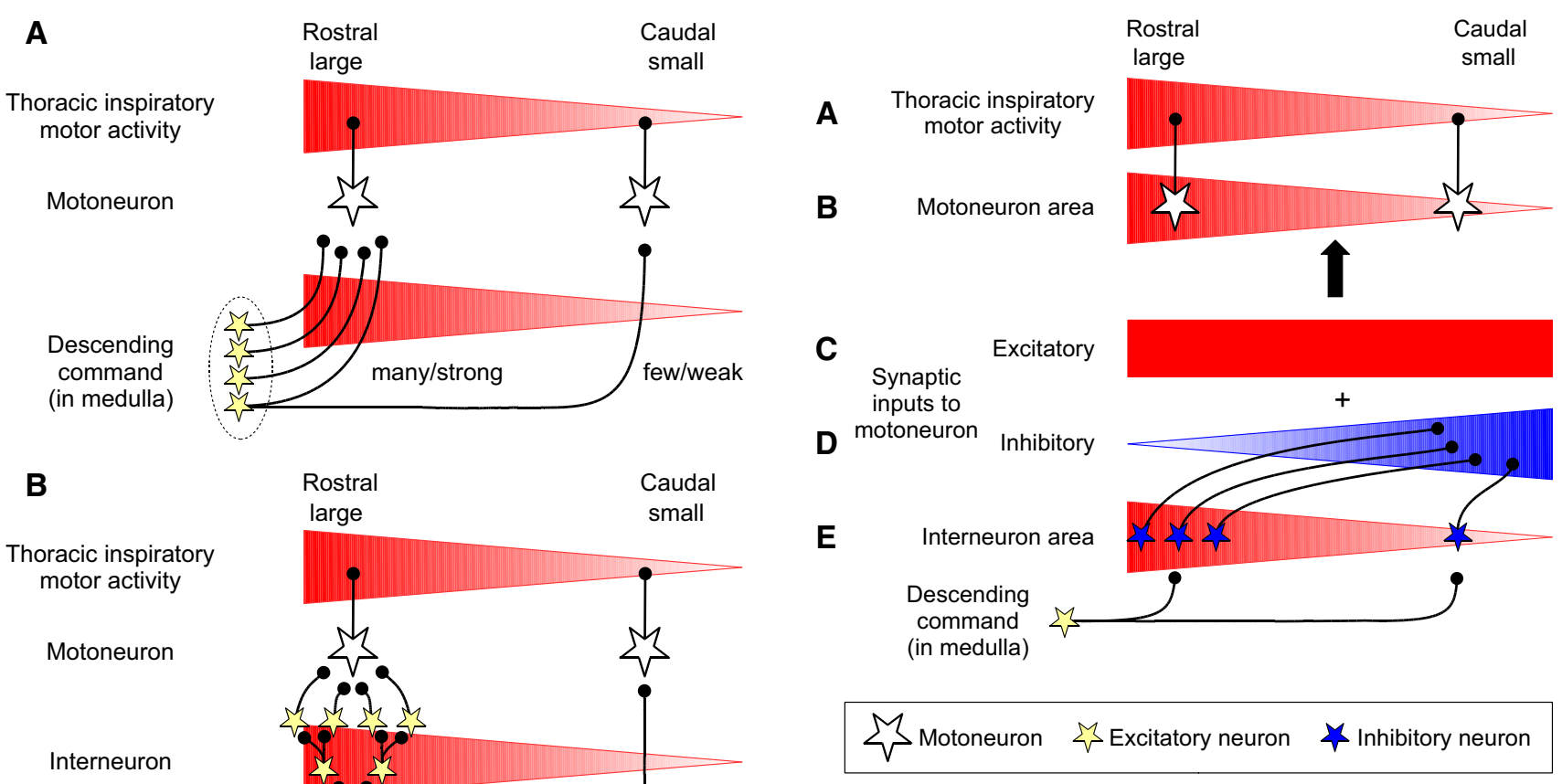

Fig. 9 a Possible neuronal mechanism in which inhibitory spinal interneurons are involved in the rostrocaudal gradient of the inspiratory motor activity. The inspiratory depolarizing optical signals in the motoneuron and interneuron areas of the rostral thoracic segments are larger than those in the caudal thoracic segments (b, e) [101]. Many of the thoracic respiratory interneurons had an axon descending a few segments, which would be inhibitory [97, 100]. Based on these studies, it is possible that the inhibitory synaptic inputs to motoneurons gradually increase to reach the caudal segments (d). In order for the motoneurons to be activated during the inspiratory phase, it is necessary to receive excitatory synaptic inputs (c). Thus, in this model, the combination of inhibitory and excitatory synaptic inputs to motoneurons forms the rostrocaudal gradient of the thoracic inspiratory motor activity

Fig. 8 The possible neuronal mechanisms underlying the organizing pattern wherein the inspiratory motor activities in the rostral thoracic segments are larger than those in the caudal thoracic segments. a The pattern is organized at the level of medulla where descending neurons exist. The descending neurons project richly to the motoneurons positioned at more rostral thoracic cord. b An example showing that the pattern is organized at the level of the spinal cord. In this example, the number of inspiratory excitatory interneurons is larger than that in the rostral segments, and these neurons amplify the excitatory inputs to the motoneurons in the rostral segments

bulbospinal neurons have systematic patterns of connections to different segments. Thus, the first and second ways mentioned above would be unlikely. Related to the third way, to the best of our knowledge, no studies have shown any differences in the membrane potentials or thresholds of the motoneurons in different thoracic segments. In summary, it is unlikely that the rostrocaudal gradient is organized entirely by the bulbospinal neurons and motoneurons.

Extracellular and intracellular recordings have shown that many thoracic interneurons have respiratory activity [97-100]. The interneurons that project to the thoracic ventral horn are mainly distributed in the contralateral medial ventral horn in the same spinal segment [99]. Morphological studies of the thoracic respiratory interneurons have shown the terminations of their collaterals are located in the ventral horn or the intermediate area

[100]. In all of these electrophysiological studies, however, the recordings were obtained from restrictive thoracic segments, and it is impossible to know the rostrocaudal distribution of the inspiratory interneurons. Using a voltage-sensitive dye, Iizuka et al. [101] demonstrated that the interneuron area of the ventral surface of the spinal cord at the more rostral thoracic segments showed larger depolarizing optical signals during the inspiratory phase (Fig. 9e). This implies that the number of inspiratory interneurons is larger in the more rostral thoracic segments and/or that the inspiratory excitatory postsynaptic potentials are larger in the interneurons that are positioned at the more rostral segments.

Regarding the contribution of these inspiratory interneurons in the rostrocaudal gradient of the inspiratory motor activity, a simple hypothesis is that these interneurons are excitatory and that they directly or indirectly provide excitatory synaptic inputs to the inspiratory motoneurons at their adjacent segments (Fig. 8b). However, one of the major characteristics of the thoracic respiratory interneurons is that many of them have an axon 
projecting to the contralateral side and descending a few segments [97, 100]. Extracellular recordings from the respiratory interneurons in this area and spike-triggered averaging were used to examine the existence of focal synaptic potentials in the contralateral thoracic ventral horn $[98,99]$. When the firing of the strongly modulated phasic inspiratory or expiratory interneurons was used as a trigger, the focal synaptic potentials that were obtained were positive in most cases; thus, these interneurons would be inhibitory [99]. The same study showed that weakly modulated tonic respiratory interneurons would be excitatory, but that their field potentials were generally small. Taken together, these findings suggest that the inhibitory inspiratory interneurons are involved in the generation of the rostrocaudal gradient of inspiratory motor activity. If this is the case, it is possible that the inspiratory inhibitory interneurons in the rostral thoracic segments project their axons to the caudal thoracic segments, and form a gradient that is the inverse of the inhibitory synaptic inputs to the motoneurons. Thus, the inhibitory synaptic inputs to the motoneurons are larger in the motoneurons that are positioned at the more caudal thoracic segments (Fig. 9). Intracellular recordings from the thoracic motoneurons of the rat have shown that some motoneurons receive a complex combination of excitatory and inhibitory synaptic inputs in the same respiratory phase [102]. Further study to examine whether the inspiratory motoneurons that are positioned at the more caudal thoracic segments receive larger inhibitory synaptic inputs during the inspiratory phase is necessary to confirm the possibility shown in Fig. 9.

In conclusion, the central respiratory networks organize the rostrocaudal gradient of the intercostal inspiratory motor activity. It seemed that the spinal inspiratory interneurons are involved in the generation of the rostrocaudal gradient. Since these neuronal mechanisms are kept intact in isolated brainstem-spinal cord preparations from the neonatal rat $[88,95]$, this in vitro preparation would be an excellent experimental model to examine the involvement of the spinal interneurons.

\section{Section 6: The functional involvement of the pons in the respiratory control mechanisms $(H$. Onimaru \& H. Koizumi)}

The pons, which is traditionally referred to as the pneumotaxic center [103], is known to be critically involved in the control of respiration; however, its functional role in respiratory rhythm and pattern generation has not been fully established. The pontine respiratory regions include the Kölliker-Fuse nucleus (KF) and the parabrachial complex (PB) in the dorsolateral pons, which are assumed to be the most important regions in the regulation of respiratory activity and respiratory phase transition [104-107], as well as several areas in the ventrolateral pons. These pontine structures interact with multiple medullary respiratory centers and regulate respiratory activity [104]. For instance, the A5 noradrenergic neurons are presumed to send inhibitory synaptic inputs to the respiratory rhythm generators in the medulla [108, 109]. Moreover, it has been demonstrated that the neurons of the locus coeruleus receive synaptic inhibition from respiratory neurons $[110,111]$, and that the electrical stimulation of the PB causes the termination of the inspiratory burst discharge and respiratory phase transition [105]. In a previous optical recording study in en bloc preparation from newborn rats, strong respiratory activity was found to be present in the KF region [112]. Respiratory activity, although weaker than that in the KF region, was also detected in the PB region. In addition, whole-cell recordings demonstrated that several types of respiratory-related neurons were located in the KF [105, 112]. In experiments using perfused brainstem preparation from juvenile rats, the KF was shown to primarily gate the respiratory motor activity of the cranial nerves innervating the laryngeal adductor and tongue muscles and the KF is thought to have a physiological role in the coordination of sequential pharyngeal swallowing with respiration [106, 107, 113].

In the newborn brainstem-spinal cord preparation from newborn rats, the medulla (without the pons), was capable of generating a three- or four-phase respiratory pattern: pre-I, inspiratory, post-I and late E $[9,114,115]$. On the other hand, in in situ arterially perfused brainstem-spinal cord preparation from adult rats, the pontine structures are necessary for the generation of the normal three-phase respiratory pattern: inspiratory, post-I, and late E [56], similar to that which is recorded in vivo $[57,116]$. A series of sequential rostrocaudal microtransections through the brainstem demonstrated the dynamics of the transformation/reorganization of the pontine-medullary respiratory network [56]. The three-phase rhythm was only detected in the intact preparation, whereas two-phase rhythm without the post-I phase emerged after the removal of the pons. These results, along with those of previous studies, suggest that the inputs from the pontine circuits shape the respiratory pattern through the activation of the post-I neurons and the inspiratory off-switch mechanisms [105, 106].

In conclusion, although different types of experimental preparations showed the various motor output patterns of respiratory activity, recent studies have demonstrated the significant roles of the pons in the formation of respiratory burst patterns and in the control of respiratory phase transitions and respiratory reflexes [56, 117-120]. We propose that the pons, interacting with the medullary respiratory 
circuits, has important roles in controlling the various physiological and pathophysiological respiration-related behaviors.

\section{Conclusions}

The understanding of the physiological and anatomical mechanisms underlying respiratory control has been achieved through the development of various technologies. In addition to electrophysiological analyses, optical imaging studies have facilitated the discovery of most of the regions in the lower brainstem and spinal cord that are involved in respiratory rhythm and pattern formation. The genetic approach has also revealed the neurophysiological and neuroanatomical mechanisms. Medullary respiratory rhythm generators $\mathrm{pFRG}$ and preBötC are described in both anatomy and physiology. The primary rhythms are modulated by various regions in the brainstem including the pons. The respiratory motor activities are then formed through premotor and motor-efferent networks including interneurons in the brainstem and spinal cord. The upper cervical spinal cord is involved in shaping eupneic breathing patterns. We believe that our current knowledge heralds the promise of further advances in the understanding of the integration of the respiratory control mechanisms in the pons, medulla, and spinal cord.

Acknowledgments This work was supported by JSPS KAKENHI Grant Numbers 25540130, 26460311, 26670676, and 15K00417 to Y. Okada, and by a grant from the Naito Foundation to K.I., and MEXT (Ministry of Education, Culture, Sports, Science and Technology)Supported Program for the Subsidies for Private Universities (Showa University of Medicine, Jichi Medical University, Hyogo College of Medicine).

\section{Compliance with ethical standards}

Conflict of interest The authors declare no conflicts of interest in association with this study.

\section{References}

1. Onimaru H, Arata A, Homma I (1995) Intrinsic burst generation of preinspiratory neurons in the medulla of brainstem-spinal cord preparations isolated from newborn rats. Exp Brain Res 106:57-68

2. Smith JC, Morrison DE, Ellenberger HH, Otto MR, Feldman JL (1989) Brainstem projections to the major respiratory neuron populations in the medulla of the cat. J Comp Neurol 281:69-96

3. Ellenberger HH, Feldman JL (1990) Brainstem connections of the rostral ventral respiratory group of the rat. Brain Res 513:35-42

4. Ballanyi K, Ruangkittisakul A, Onimaru H (2009) Opioids prolong and anoxia shortens delay between onset of preinspiratory (pFRG) and inspiratory (preBötC) network bursting in newborn rat brainstems. Pflugers Arch 458:571-587
5. Ezure K (2004) Reflections on respiratory rhythm generation. Prog Brain Res 143:67-74

6. Duffin J (2004) Functional organization of respiratory neurones: a brief review of current questions and speculations. Exp Physiol 89:517-529

7. Onimaru H, Arata A, Homma I (1987) Localization of respiratory rhythm-generating neurons in the medulla of brainstemspinal cord preparations from newborn rats. Neurosci Lett 78:151-155

8. Arata A, Onimaru H, Homma I (1990) Respiration-related neurons in the ventral medulla of newborn rats in vitro. Brain Res Bull 24:599-604

9. Ballanyi K, Onimaru H, Homma I (1999) Respiratory network function in the isolated brainstem-spinal cord of newborn rats. Prog Neurobiol 59:583-634

10. Onimaru H, Ikeda K, Kawakami K (2008) CO2-sensitive preinspiratory neurons of the parafacial respiratory group express Phox $2 b$ in the neonatal rat. J Neurosci 28:12845-12850

11. Onimaru H, Ikeda K, Kawakami K (2009) Phox2b, RTN/pFRG neurons and respiratory rhythmogenesis. Respir Physiol Neurobiol 168:13-18

12. Onimaru H, Dutschmann M (2012) Calcium imaging of neuronal activity in the most rostral parafacial respiratory group of the newborn rat. J Physiol Sci 62:71-77

13. Thoby-Brisson M, Karlen M, Wu N, Charnay P, Champagnat J, Fortin G (2009) Genetic identification of an embryonic parafacial oscillator coupling to the preBötzinger complex. Nat Neurosci 12:1028-1035

14. Guyenet PG, Stornetta RL, Bayliss DA (2008) Retrotrapezoid nucleus and central chemoreception. J Physiol 586:20432048

15. Kang BJ, Chang DA, Mackay DD, West GH, Moreira TS, Takakura AC, Gwilt JM, Guyenet PG, Stornetta RL (2007) Central nervous system distribution of the transcription factor Phox $2 b$ in the adult rat. J Comp Neurol 503:627-641

16. Stornetta RL, Moreira TS, Takakura AC, Kang BJ, Chang DA, West GH, Brunet JF, Mulkey DK, Bayliss DA, Guyenet PG (2006) Expression of Phox2b by brainstem neurons involved in chemosensory integration in the adult rat. $\mathrm{J}$ Neurosci 26:10305-10314

17. Dubreuil V, Ramanantsoa N, Trochet D, Vaubourg V, Amiel J, Gallego J, Brunet JF, Goridis C (2008) A human mutation in Phox $2 b$ causes lack of $\mathrm{CO} 2$ chemosensitivity, fatal central apnea, and specific loss of parafacial neurons. Proc Natl Acad Sci USA 105:1067-1072

18. Abbott SB, Stornetta RL, Coates MB, Guyenet PG (2011) Phox $2 b$-expressing neurons of the parafacial region regulate breathing rate, inspiration, and expiration in conscious rats. J Neurosci 31:16410-16422

19. Pagliardini S, Janczewski WA, Tan W, Dickson CT, Deisseroth K, Feldman JL (2011) Active expiration induced by excitation of ventral medulla in adult anesthetized rats. J Neurosci 31:2895-2905

20. Huckstepp RT, Cardoza KP, Henderson LE, Feldman JL (2015) Role of parafacial nuclei in control of breathing in adult rats. J Neurosci 35:1052-1067

21. Onimaru H, Homma I (2003) A novel functional neuron group for respiratory rhythm generation in the ventral medulla. J Neurosci 23:1478-1486

22. Mellen NM, Janczewski WA, Bocchiaro CM, Feldman JL (2003) Opioid-induced quantal slowing reveals dual networks for respiratory rhythm generation. Neuron 37:821-826

23. Janczewski WA, Onimaru H, Homma I, Feldman JL (2002) Opioid-resistant respiratory pathway from the preinspiratory neurones to abdominal muscles: in vivo and in vitro study in the newborn rat. J Physiol 545:1017-1026 
24. Onimaru H, Ikeda K, Kawakami K (2012) Postsynaptic mechanisms of $\mathrm{CO}(2)$ responses in parafacial respiratory neurons of newborn rats. J Physiol 590:1615-1624

25. Onimaru H, Ikeda K, Kawakami K (2012) Relationship between the distribution of the paired-like homeobox gene (Phox $2 b$ ) expressing cells and blood vessels in the parafacial region of the ventral medulla of neonatal rats. Neuroscience 212:131-139

26. Onimaru H, Ikeda K, Mariho T, Kawakami K (2014) Cytoarchitecture and $\mathrm{CO}(2)$ sensitivity of Phox2b-positive Parafacial neurons in the newborn rat medulla. Prog Brain Res 209:57-71

27. Amiel J, Laudier B, Attié-Bitach T, Trang H, de Pontual L, Gener B, Trochet D, Etchevers H, Ray P, Simonneau M, Vekemans M, Munnich A, Gaultier C, Lyonnet S (2003) Polyalanine expansion and frameshift mutations of the pairedlike homeobox gene PHOX2B in congenital central hypoventilation syndrome. Nat Genet 33:459-461

28. Ikeda K, Takahashi M, Sato S, Igarashi H, Ishizuka T, Yawo H, Arata S, Southard-Smith EM, Kawakami K, Onimaru H (2015) A Phox 2b BAC transgenic rat line useful for understanding respiratory rhythm generator neural circuitry. PLoS One 10:e0132475

29. Rudzinski E, Kapur RP (2010) PHOX2B immunolocalization of the candidate human retrotrapezoid nucleus. Pediatr Dev Pathol 13:291-299

30. Smith JC, Ellenberger HH, Ballanyi K, Richter DW, Feldman JL (1991) Pre-Bötzinger complex: a brainstem region that may generate respiratory rhythm in mammals. Science 254:726-729

31. Ruangkittisakul A, Schwarzacher SW, Secchia L, Poon BY, Ma Y, Funk GD, Ballanyi K (2006) High sensitivity to neuromodulator-activated signaling pathways at physiological $[\mathrm{K}+]$ of confocally imaged respiratory center neurons in on-line-calibrated newborn rat brainstem slices. J Neurosci 26:11870-11880

32. Ruangkittisakul A, Kottick A, Picardo MC, Ballanyi K, Del Negro CA (2014) Identification of the pre-Bötzinger complex inspiratory center in calibrated "sandwich" slices from newborn mice with fluorescent Dbx1 interneurons. Physiol Rep 2(e12111):1-16

33. Krause KL, Forster HV, Kiner T, Davis SE, Bonis JM, Qian B, Pan LG (2009) Normal breathing pattern and arterial blood gases in awake and sleeping goats after near total destruction of the presumed pre-Bötzinger complex and the surrounding region. J Appl Physiol 1985 106:605-619

34. Schwarzacher SW, Rub U, Deller T (2011) Neuroanatomical characteristics of the human pre-Bötzinger complex and its involvement in neurodegenerative brainstem diseases. Brain $134: 24-35$

35. Koshiya N, Smith JC (1999) Neuronal pacemaker for breathing visualized in vitro. Nature 400:360-363

36. Koshiya N, Oku Y, Yokota S, Oyamada Y, Yasui Y, Okada Y (2014) Anatomical and functional pathways of rhythmogenic inspiratory premotor information flow originating in the preBötzinger complex in the rat medulla. Neuroscience 268:194-211

37. Kuwana S, Tsunekawa N, Yanagawa Y, Okada Y, Kuribayashi J, Obata K (2006) Electrophysiological and morphological characteristics of GABAergic respiratory neurons in the mouse pre-Bötzinger complex. Eur J Neurosci 23:667-674

38. Gray PA, Janczewski WA, Mellen N, McCrimmon DR, Feldman JL (2001) Normal breathing requires preBötzinger complex neurokinin-1 receptor-expressing neurons. Nat Neurosci 4:927-930

39. Gray PA, Hayes JA, Ling GY, Llona I, Tupal S, Picardo MC, Ross SE, Hirata T, Corbin JG, Eugenin J, Del Negro CA (2010) Developmental origin of preBötzinger complex respiratory neurons. J Neurosci 30:14883-14895
40. Wang H, Stornetta RL, Rosin DL, Guyenet PG (2001) Neurokinin-1 receptor-immunoreactive neurons of the ventral respiratory group in the rat. J Comp Neurol 434:128-146

41. Stornetta RL, Rosin DL, Wang H, Sevigny CP, Weston MC, Guyenet PG (2003) A group of glutamatergic interneurons expressing high levels of both neurokinin-1 receptors and somatostatin identifies the region of the pre-Bötzinger complex. J Comp Neurol 455:499-512

42. Wei XY, Zhao Y, Wong-Riley MT, Ju G, Liu YY (2012) Synaptic relationship between somatostatin- and neurokinin-1 receptor-immunoreactive neurons in the pre-Bötzinger complex of rats. J Neurochem 122:923-933

43. Wang X, Hayes JA, Revill AL, Song H, Kottick A, Vann NC, LaMar MD, Picardo MC, Akins VT, Funk GD, Del Negro CA (2014) Laser ablation of Dbx1 neurons in the pre-Bötzinger complex stops inspiratory rhythm and impairs output in neonatal mice. Elife 3:e03427

44. Okada Y, Sasaki T, Oku Y, Takahashi N, Seki M, Ujita S, Tanaka KF, Matsuki N, Ikegaya Y (2012) Preinspiratory calcium rise in putative pre-Bötzinger complex astrocytes. J Physiol 590:4933-4944

45. Oku Y, Fresemann J, Miwakeichi F, Hulsmann S (2016) Respiratory calcium fluctuations in low-frequency oscillating astrocytes in the pre-Bötzinger complex. Respir Physiol Neurobiol 226:11-17

46. Liu YY, Wong-Riley MT, Liu JP, Wei XY, Jia Y, Liu HL, Fujiyama F, Ju G (2004) Substance P and enkephalinergic synapses onto neurokinin-1 receptor-immunoreactive neurons in the pre-Bötzinger complex of rats. Eur J Neurosci 19:65-75

47. Tan W, Pagliardini S, Yang P, Janczewski WA, Feldman JL (2010) Projections of preBötzinger complex neurons in adult rats. J Comp Neurol 518:1862-1878

48. Dobbins EG, Feldman JL (1994) Brainstem network controlling descending drive to phrenic motoneurons in rat. J Comp Neurol 347:64-86

49. Oka T, Yokota S, Tsumori T, Niu JG, Yasui Y (2012) Glutamatergic neurons in the lateral periaqueductal gray innervate neurokinin-1 receptor-expressing neurons in the ventrolateral medulla of the rat. Neurosci Res 74:106-115

50. Johnson SM, Smith JC, Funk GD, Feldman JL (1994) Pacemaker behavior of respiratory neurons in medullary slices from neonatal rat. J Neurophysiol 72:2598-2608

51. Pagliardini S, Adachi T, Ren J, Funk GD, Greer JJ (2005) Fluorescent tagging of rhythmically active respiratory neurons within the pre-Bötzinger complex of rat medullary slice preparations. J Neurosci 25:2591-2596

52. Del Negro CA, Koshiya N, Butera RJ Jr, Smith JC (2002) Persistent sodium current, membrane properties and bursting behavior of pre-bötzinger complex inspiratory neurons in vitro. J Neurophysiol 88:2242-2250

53. Koizumi H, Smith JC (2008) Persistent Na+ and K+-dominated leak currents contribute to respiratory rhythm generation in the pre-Bötzinger complex in vitro. J Neurosci 28:1773-1785

54. Butera RJ Jr, Rinzel J, Smith JC (1999) Models of respiratory rhythm generation in the pre-Bötzinger complex. I. Bursting pacemaker neurons. J Neurophysiol 82:382-397

55. Koizumi H, Koshiya N, Chia JX, Cao F, Nugent J, Zhang R, Smith JC (2013) Structural-functional properties of identified excitatory and inhibitory interneurons within pre-Bötzinger complex respiratory microcircuits. J Neurosci 33:2994-3009

56. Smith JC, Abdala AP, Koizumi H, Rybak IA, Paton JF (2007) Spatial and functional architecture of the mammalian brain stem respiratory network: a hierarchy of three oscillatory mechanisms. J Neurophysiol 98:3370-3387

57. Paton JF, Abdala AP, Koizumi H, Smith JC, St-John WM (2006) Respiratory rhythm generation during gasping depends on persistent sodium current. Nat Neurosci 9:311-313 
58. Koizumi H, Smerin SE, Yamanishi T, Moorjani BR, Zhang R, Smith JC (2010) TASK channels contribute to the K+-dominated leak current regulating respiratory rhythm generation in vitro. J Neurosci 30:4273-4284

59. Thoby-Brisson M, Ramirez JM (2001) Identification of two types of inspiratory pacemaker neurons in the isolated respiratory neural network of mice. J Neurophysiol 86:104-112

60. Del Negro CA, Morgado-Valle C, Hayes JA, Mackay DD, Pace RW, Crowder EA, Feldman JL (2005) Sodium and calcium current-mediated pacemaker neurons and respiratory rhythm generation. J Neurosci 25:446-453

61. Del Negro CA, Johnson SM, Butera RJ, Smith JC (2001) Models of respiratory rhythm generation in the pre-Bötzinger complex. III. Experimental tests of model predictions. J Neurophysiol 86:59-74

62. Carroll MS, Ramirez JM (2012) Cycle-by-cycle assembly of respiratory network activity is dynamic and stochastic. J Neurophysiol 109:296-305

63. Koizumi H, Wilson CG, Wong S, Yamanishi T, Koshiya N, Smith JC (2008) Functional imaging, spatial reconstruction, and biophysical analysis of a respiratory motor circuit isolated in vitro. J Neurosci 28:2353-2365

64. Morgado-Valle C, Baca SM, Feldman JL (2010) Glycinergic pacemaker neurons in preBötzinger complex of neonatal mouse. J Neurosci 30:3634-3639

65. Johnson SM, Koshiya N, Smith JC (2001) Isolation of the kernel for respiratory rhythm generation in a novel preparation: the preBötzinger complex "island". J Neurophysiol 85:1772-1776

66. Davies JG, Kirkwood PA, Sears TA (1985) The distribution of monosynaptic connexions from inspiratory bulbospinal neurones to inspiratory motoneurones in the cat. J Physiol 368:63-87

67. Lipski J, Duffin J (1986) An electrophysiological investigation of propriospinal inspiratory neurons in the upper cervical cord of the cat. Exp Brain Res 61:625-637

68. Aoki M, Kasaba T, Kurosawa Y, Ohtsuka K, Satomi H (1984) The projection of cervical respiratory neurons to the phrenic nucleus in the cat. Neurosci Lett Suppl 17:S49

69. Nakazono Y, Aoki M (1994) Excitatory connections between upper cervical inspiratory neurons and phrenic motoneurons in cats. J Appl Physiol (1985) 77:679-683

70. Illert M, Lundberg A, Padel Y, Tanaka R (1978) Integration in descending motor pathways controlling the forelimb in the cat. 5. Properties of and monosynaptic excitatory convergence on C3-C4 propriospinal neurones. Exp Brain Res 33:101-130

71. Palisses R, Perségol L, Viala D (1989) Evidence for respiratory interneurones in the C3-C5 cervical spinal cord in the decorticate rabbit. Exp Brain Res 78:624-632

72. Aoki M, Mori S, Kawahara K, Watanabe H, Ebata N (1980) Generation of spontaneous respiratory rhythm in high spinal cats. Brain Res 202:51-63

73. Coglianese CJ, Peiss CN, Wurster RD (1977) Rhythmic phrenic nerve activity and respiratory activity in spinal dogs. Respir Physiol 29:247-254

74. Viala D, Freton E (1983) Evidence for respiratory and locomotor pattern generators in the rabbit cervico-thoracic cord and for their interactions. Exp Brain Res 49:247-256

75. Dubayle D, Viala D (1996) Localization of the spinal respiratory rhythm generator by an in vitro electrophysiological approach. NeuroReport 7:1175-1180

76. Morin D, Bonnot A, Ballion B, Viala D (2000) alpha1-adrenergic receptor-induced slow rhythmicity in nonrespiratory cervical motoneurons of neonatal rat spinal cord. Eur J Neurosci 12:2950-2966

77. Kobayashi S, Fujito Y, Matsuyama K, Aoki M (2010) Spontaneous respiratory rhythm generation in in vitro upper cervical slice preparations of neonatal mice. J Physiol Sci 60:303-307
78. Onimaru H, Ballanyi K, Homma I (2003) Contribution of Ca2+dependent conductances to membrane potential fluctuations of medullary respiratory neurons of newborn rats in vitro. J Physiol 552:727-741

79. Oku Y, Okabe A, Hayakawa T, Okada Y (2008) Respiratory neuron group in the high cervical spinal cord discovered by optical imaging. NeuroReport 19:1739-1743

80. Okada Y, Yokota S, Shinozaki Y, Aoyama R, Yasui Y, Ishiguro M, Oku Y (2009) Anatomical architecture and responses to acidosis of a novel respiratory neuron group in the high cervical spinal cord (HCRG) of the neonatal rat. Adv Exp Med Biol 648:387-394

81. Jones SE, Saad M, Lewis DI, Subramanian HH, Dutschmann M (2012) The nucleus retroambiguus as possible site for inspiratory rhythm generation caudal to obex. Respir Physiol Neurobiol 180:305-310

82. Alilain WJ, Li X, Horn KP, Dhingra R, Dick TE, Herlitze S, Silver J (2008) Light-induced rescue of breathing after spinal cord injury. J Neurosci 28:11862-11870

83. Alilain WJ, Horn KP, Hu H, Dick TE, Silver J (2011) Functional regeneration of respiratory pathways after spinal cord injury. Nature 475:196-200

84. Kirkwood PA, Sears TA, Stagg D, Westgaard RH (1982) The spatial distribution of synchronization of intercostal motoneurones in the cat. J Physiol 327:137-155

85. Greer JJ, Martin TP (1990) Distribution of muscle fiber types and EMG activity in cat intercostal muscles. J Appl Physiol (1985) 69:1208-1211

86. Le Bars P, Duron B (1984) Are the external and internal intercostal muscles synergist or antagonist in the cat? Neurosci Lett $51: 383-386$

87. De Troyer A, Ninane V (1986) Respiratory function of intercostal muscles in supine dog: an electromyographic study. J Appl Physiol (1985) 60:1692-1699

88. Legrand A, De Troyer A (1999) Spatial distribution of external and internal intercostal activity in dogs. J Physiol 518:291-300

89. De Troyer A, Gorman RB, Gandevia SC (2003) Distribution of inspiratory drive to the external intercostal muscles in humans. J Physiol 546:943-954

90. De Troyer A, Kirkwood PA, Wilson TA (2005) Respiratory action of the intercostal muscles. Physiol Rev 85:717-756

91. Legrand A, Brancatisano A, Decramer M, De Troyer A (1996) Rostrocaudal gradient of electrical activation in the parasternal intercostal muscles of the dog. J Physiol 495(Pt 1):247-254

92. Gandevia SC, Hudson AL, Gorman RB, Butler JE, De Troyer A (2006) Spatial distribution of inspiratory drive to the parasternal intercostal muscles in humans. J Physiol 573:263-275

93. Iizuka M (2004) Rostrocaudal distribution of spinal respiratory motor activity in an in vitro neonatal rat preparation. Neurosci Res 50:263-269

94. Burke RE, Dum RP, Fleshman JW, Glenn LL, Lev-Tov A, O'Donovan MJ, Pinter MJ (1982) A HRP study of the relation between cell size and motor unit type in cat ankle extensor motoneurons. J Comp Neurol 209:17-28

95. Davies JG, Kirkwood PA, Sears TA (1985) The detection of monosynaptic connexions from inspiratory bulbospinal neurones to inspiratory motoneurones in the cat. J Physiol 368:33-62

96. Duffin J, Lipski J (1987) Monosynaptic excitation of thoracic motoneurones by inspiratory neurones of the nucleus tractus solitarius in the cat. J Physiol 390:415-431

97. Kirkwood PA, Munson JB, Sears TA, Westgaard RH (1988) Respiratory interneurones in the thoracic spinal cord of the cat. J Physiol 395:161-192

98. Kirkwood PA, Schmid K, Sears TA (1993) Functional identities of thoracic respiratory interneurones in the cat. J Physiol 461:667-687 
99. Schmid K, Kirkwood PA, Munson JB, Shen E, Sears TA (1993) Contralateral projections of thoracic respiratory interneurones in the cat. J Physiol 461:647-665

100. Saywell SA, Ford TW, Meehan CF, Todd AJ, Kirkwood PA (2011) Electrophysiological and morphological characterization of propriospinal interneurons in the thoracic spinal cord. J Neurophysiol 105:806-826

101. Iizuka M, Onimaru H, Izumizaki M (2016) Distribution of respiration-related neuronal activity in the thoracic spinal cord of the neonatal rat: an optical imaging study. Neuroscience 315:217-227

102. de Almeida AT, Kirkwood PA (2010) Multiple phases of excitation and inhibition in central respiratory drive potentials of thoracic motoneurones in the rat. J Physiol 588:2731-2744

103. Lumsden $T$ (1923) Observations on the respiratory centres. J Physiol 57:354-367

104. Martelli D, Stanic D, Dutschmann M (2013) The emerging role of the parabrachial complex in the generation of wakefulness drive and its implication for respiratory control. Respir Physiol Neurobiol 188:318-323

105. Arata A (2009) Respiratory activity of the neonatal dorsolateral pons in vitro. Respir Physiol Neurobiol 168:144-152

106. Dutschmann M, Herbert H (2006) The Kölliker-Fuse nucleus gates the postinspiratory phase of the respiratory cycle to control inspiratory off-switch and upper airway resistance in rat. Eur $\mathbf{J}$ Neurosci 24:1071-1084

107. Bautista TG, Dutschmann M (2014) Inhibition of the pontine Kölliker-Fuse nucleus abolishes eupneic inspiratory hypoglossal motor discharge in rat. Neuroscience 267:22-29

108. Hilaire G, Monteau R, Errchidi S (1989) Possible modulation of the medullary respiratory rhythm generator by the noradrenergic A5 area: an in vitro study in the newborn rat. Brain Res 485:325-332

109. Errchidi S, Monteau R, Hilaire G (1991) Noradrenergic modulation of the medullary respiratory rhythm generator in the newborn rat: an in vitro study. J Physiol 443:477-498
110. Oyamada Y, Ballantyne D, Muckenhoff K, Scheid P (1998) Respiration-modulated membrane potential and chemosensitivity of locus coeruleus neurones in the in vitro brainstem-spinal cord of the neonatal rat. J Physiol 513(Pt 2):381-398

111. Onimaru H, Homma I (2005) Optical imaging of respiratory neuron activity from the dorsal view of the lower brainstem. Clin Exp Pharmacol Physiol 32:297-301

112. Kobayashi S, Onimaru H, Inoue M, Inoue T, Sasa R (2005) Localization and properties of respiratory neurons in the rostral pons of the newborn rat. Neuroscience 134:317-325

113. Bautista TG, Dutschmann M (2014) Ponto-medullary nuclei involved in the generation of sequential pharyngeal swallowing and concomitant protective laryngeal adduction in situ. J Physiol 592:2605-2623

114. Arata A, Onimaru H, Homma I (1998) Possible synaptic connections of expiratory neurons in the medulla of newborn rat in vitro. NeuroReport 9:743-746

115. Onimaru H, Arata A, Homma I (1997) Neuronal mechanisms of respiratory rhythm generation: an approach using in vitro preparation. Jpn J Physiol 47:385-403

116. St-John WM, Paton JF (2003) Defining eupnea. Respir Physiol Neurobiol 139:97-103

117. Alheid GF, Milsom WK, McCrimmon DR (2004) Pontine influences on breathing: an overview. Respir Physiol Neurobiol 143:105-114

118. Cohen MI, Shaw CF (2004) Role in the inspiratory off-switch of vagal inputs to rostral pontine inspiratory-modulated neurons. Respir Physiol Neurobiol 143:127-140

119. Okazaki M, Takeda R, Yamazaki H, Haji A (2002) Synaptic mechanisms of inspiratory off-switching evoked by pontine pneumotaxic stimulation in cats. Neurosci Res 44:101-110

120. Song G, Poon CS (2004) Functional and structural models of pontine modulation of mechanoreceptor and chemoreceptor reflexes. Respir Physiol Neurobiol 143:281-292 